\title{
Turbulence in the Lower Troposphere: Second-Order Closure and Mass-Flux Modelling Frameworks
}

\author{
Dmitrii V. Mironov
}

Deutscher Wetterdienst, Forschung und Entwicklung, FE14, Kaiserleistr. 29/35, D-63067 Offenbach am Main, Germany. dmitrii.mironov@dwd.de

\section{Introduction}

Turbulence is ubiquitous in the earth's atmosphere. Its spatial scales range from many tens of kilometres to a few millimetres. A wide assortment of turbulent eddies of different size and shape lies within this range. These include breeze circulations, storms, clouds, plumes and rolls in the planetary boundary layer $(\mathrm{PBL})$, and eddies in urban street canyons and in plant canopies, to mention a few. It is this large variety of turbulent motions that numerical weather prediction (NWP) models, as well as other numerical models of the atmosphere, have to deal with.

Atmospheric turbulence is a notoriously difficult and extensive subject. Even a cursory examination of its most important aspects would require a voluminous account that goes far beyond the scope of the present paper. We restrict our consideration to modelling turbulence in the lower troposphere as it is practised nowadays in NWP and related applications, e.g. climate modelling and air pollution dispersion studies. Turbulence modelling in this context means the representation of the effect of turbulent motions, which are not explicitly computed by a numerical model of the atmosphere, on the explicitly computed fields. Other topics, such as measurements and numerical simulations (large-eddy and direct numerical simulations) of turbulence in the atmosphere, although very important, are not considered here. Readers are referred to review articles and books $[132,184,66,209,60,120,160,68,99$, $67,61]$, where further references can be found.

Before proceeding any further, we recall a plain point that a numerical model of the atmosphere solves the evolution equations in the form

$$
\frac{\partial\langle f\rangle}{\partial t}+\left\langle u_{i}\right\rangle \frac{\partial\langle f\rangle}{\partial x_{i}}=-\frac{\partial\left\langle u_{i}^{\prime} f^{\prime}\right\rangle}{\partial x_{i}}+F_{f}
$$

where $t$ is the time, $x_{i}$ are the space co-ordinates, and $u_{i}$ are the velocity components. The Einstein summation convention for repeated indices is adopted. 
A generic variable $f$ refers to any quantity treated by an atmospheric model, and $F_{f}$ symbolises the source of $f$ due to various processes, such as radiation and precipitation. The angle brackets denote the quantities that are explicitly computed (resolved) by a numerical model, and primes denote deviations therefrom. The incompressibility is assumed which is a fairly accurate approximation for the lower troposphere. Equation (1) is obtained by applying a spatial filter to the governing momentum and scalar equations (see chapter 7 of this volume). The quantity $\left\langle u_{i}^{\prime} f^{\prime}\right\rangle$ represents the flux of $f$ due to sub-filter scale motions. ${ }^{1}$ In what follows, the sub-filter scale quantities will be referred to, perhaps somewhat loosely, as the sub-grid scale (SGS) quantities, considering that the latter term has been universally accepted. As the SGS motions are not explicitly computed by a numerical model, the SGS flux must be modelled, or parameterised, in terms of resolved quantities. The terms "model" and "parameterisation scheme" may be used interchangeably in this context. The term "parameterisation scheme" is more often used in the NWP community.

The SGS flux divergence term on the right-hand side (r.h.s.) of Eq. (1) should in principle represent the effect of all SGS motions down to the smallest scales where turbulence kinetic energy (TKE) eventually dissipates. It is, however, customary for NWP models to split this term into contributions due to various processes. The contributions due to "turbulence", that is thought to represent quasi-random small-scale motions, and due to "convection", that is thought to represent quasi-organised motions of larger scales, are usually distinguished. That is,

$$
\left\langle u_{i}^{\prime} f^{\prime}\right\rangle=\left\langle u_{i}^{\prime} f^{\prime}\right\rangle_{\text {turb }}+\left\langle u_{i}^{\prime} f^{\prime}\right\rangle_{\text {conv }} .
$$

The SGS momentum flux may also contain a contribution due to the orographic drag, i.e. the unresolved drag caused by the effects of sub-grid scale orography, such as the absorption and reflection of orographically induced gravity waves. The orographic drag parameterisation issues will not be considered in what follows. Readers are referred to [36, 140, 130, 23, 186], where further references can be found. Although the decomposition (2) is commonly accepted, it is not as innocent as it might seem. Caution is required to ensure that the sum of the above two contributions actually represents the total SGS flux. Otherwise, serious problems may be encountered, for example, doublecounting of some energetically relevant modes of SGS motions, or their loss.

It is general practice in NWP and related applications to model the two contributions on the r.h.s. of Eq. (2) in different ways. Turbulence parameterisation schemes are usually developed on the basis of the ensemblemean second-order turbulence closure approach. Convection parameterisation

${ }^{1}$ Strictly speaking, the Lilly [124] notation with no primes should be used to emphasise that the filter does not necessarily satisfy the Reynolds averaging assumptions. The sub-filter scale flux is then given by $\left\langle u_{i} f\right\rangle-\left\langle u_{i}\right\rangle\langle f\rangle$. See $[184,110]$ for further discussion. 
schemes are usually developed on the basis of the mass-flux approach. In the subsequent text (sections 2 and 3), we will consider these parameterisation schemes in some detail. We attempt to show that the two approaches have much in common, although differences remain and they may be important.

Convection in the atmosphere manifests itself in many different forms. Dry convection is usually driven by the surface buoyancy flux and is confined to the PBL. Regimes of moist convection are many and varied. As nicely stated by Stevens [188] "moist convection is many, rather than one thing." This author presents a comprehensive account of many essential features of moist atmospheric convection, including phenomenology and theoretical frameworks to describe its major regimes, namely, stratocumulus, shallow non-precipitating cumulus, and deep precipitating cumulus convection. Driven by a powerful engine - the latent heat release due to water vapour condensation in rising air parcels, moist convection can penetrate all the way up to the top of the troposphere. Such deep penetrative convection is typically associated with heavy precipitation. With their horizontal grid size of about $50 \mathrm{~km}$ or so, the present-day global NWP models are unable to resolve deep convection. A parameterisation is required to describe convective fluxes of scalar quantities and of momentum, convection source terms due to condensation/evaporation, due to release/consumption of latent heat and due to precipitation fall-out, as well as a sophisticated interplay of convective, radiative and microphysical processes. Improving deep convection parameterisations represents one of the major challenges in NWP and related applications [12]. Details of this fascinating phenomenon are discussed in [91, 60, 180,61, 188]. The present-day limited-area NWP models have a horizontal grid size of 10 to $5 \mathrm{~km}$, and there is a strong tendency to achieve an even higher resolution with a horizontal grid size of order $1 \mathrm{~km}$. With such a grid size, deep convection will likely be computed explicitly. However, the PBL turbulence and shallow convection will still remain at the sub-grid scale and will require an adequate parameterisation scheme. Some theoretical problems related to parameterising SGS motions in high-resolution atmospheric models are discussed in [211]. Notice that an increased resolution will alleviate some parameterisation issues but will make other issues even more complicated. One example is the atmospheric radiation. High-resolution NWP models will need to account for three-dimensional effects of radiation transfer, inevitably making the radiation parameterisation schemes computationally very expensive.

Noteworthy are a number of issues that complicate the development of physical parameterisations for NWP models, of turbulence-convection parameterisations in particular. Since NWP models are used operationally, their quality is judged by the quality of the final product - the weather forecast. The implementation of any innovation into an NWP model can only be justified if a new version of the NWP model that incorporates the innovation beats an older version of the model in terms of the forecast quality. This is by no means guaranteed. The NWP models are very complex non-linear systems where a very sophisticated interplay of their various components takes 
place. Apart from the physics and numerics, initialisation and data assimilation should also be borne in mind. Every component of an NWP model has its deficiencies. Working together, they produce errors that may amplify (a very unwanted situation), but may partially compensate each other as well. Then, the incorporation of a new physical parameterisation scheme does not necessarily lead to an improved forecast, no matter how advanced and physically sound that new scheme is as compared to an old scheme, i.e. to the scheme currently used in the operational version of a given NWP model. Putting it differently, it is not sufficient to prove that a new parameterisation scheme is superior to an old one in that it is more physically sound and performs well as a stand-alone physical model, e.g. as judged by a comparison of results from idealised one-dimensional experiments with observational and numerical data. It should also be ensured that a new parameterisation scheme works in harmony with the other components of a particular NWP model.

One more aspect of great significance is that in order to be useful a turbulence-convection parameterisation for NWP should be computationally efficient. Since the NWP products must be delivered to end users in due time, it is simply not possible to apply parameterisations whose high computational cost may lead to a forecast delay. There are many physically sound turbulence-convection models which proved to be very useful research tools. However, there is no way to use them in operational NWP models for they are computationally prohibitively expensive. On the other hand, a useful parameterisation scheme should account for much of the essential physics of atmospheric turbulence and convection. The key to the success in developing turbulence-convection parameterisation schemes for NWP and related applications is, therefore, to find the best possible compromise between physical realism and computational economy.

Recent advances in observations and numerical simulations of atmospheric flows along with new theoretical ideas have led to considerable progress in representing turbulence and convection in NWP models. The progress, however, is somewhat slower than one might wish. Given the severe constraints mentioned above, it seems likely that comparatively simple second-order closure schemes and mass-flux schemes will be further used in NWP models for some years to come to parameterise turbulence and convection, respectively. The question then arises whether more regime-dependent sub-models (parameterisation schemes) should be developed, or some unification of various parameterisation frameworks is possible (see discussions in $[12,188,146]$ ). A definitive answer to this question does not seem to exist at present. Some attempts have already been made to achieve a more unified description of several types of fluctuating motions (see section 4). It is also the author's opinion that a more unified description is desirable. There are several ways to do so, however, and it is not a priory clear which way should be preferred.

In the next section, we outline the ensemble-mean second-order modelling framework and briefly discuss parameterisation assumptions that should be made in order to arrive at a reasonably simple turbulence closure. As we shall 
see subsequently, only a small fraction of what is presented in sections 2.1 and 2.2 is actually used in applications. A rather extended discussion is necessary, however, in order to understand how simplified parameterisation schemes are obtained and what is lost on the way (section 2.3). In particular, comprehending the role of the third-order moments in maintaining the second-order moment budgets is the key to understanding how non-local transport properties of convective motions can be accounted for within the second-order modelling framework. A consideration of parameterisations of the pressure redistribution terms is required, among other things, to understand how the down-gradient diffusion approximation for fluxes are derived from the second-moment budget equations. Furthermore, a systematic consideration of the second-order modelling framework demonstrates the limits of applicability of simplified turbulence closures. The mass-flux parameterisation schemes are outlined in section 3. In section 3.3, we explore analogies between the ensemble-mean and the mass-flux approaches. This exercise helps to elucidate the essential physics behind various parameterisation assumptions and suggests possible ways towards their improvement. It also shows that the two approaches have much in common and suggests how the mass-flux parameterisation ideas can be translated into the language of the ensemble-mean second-order closures and vice versa. In section 4 , we discuss the steps that should be made towards an improved description of turbulence and shallow non-precipitating convection within a unified parameterisation framework. Conclusions are presented in section 5. The discussion below inevitably reflects the author's personal experience, and, to some (hopefully minor) extent, his preferences. The author apologises for omissions that are unavoidable in any effort to address such an extensive and difficult subject as atmospheric turbulence.

\section{Turbulence Parameterisation Schemes}

\subsection{Governing Equations}

The basis for the development of turbulence parameterisation schemes is the set of transport equations for the second-order turbulence moments (see e.g. [153]). Those equations are derived using the Reynolds averaging and are thought to describe the ensemble-mean statistical moments of fluctuating fields. As already noted, a filter applied to the governing momentum and scalar equations does not generally coincide with the Reynolds averaging. Hence, the sub-grid scale (sub-filter scale) moments of fluctuating fields do not coincide with the ensemble-mean moments. It is, however, assumed (often tacitly) that the two sets of moments are not too different. The ensemblemean second-order closure approach is commonly taken to parameterise the SGS fluxes and variances as if these were truly ensemble-mean quantities. Caution should be exercised since the validity of this assumption deteriorates as the resolution of atmospheric models is refined. 
For the sake of clarity, we first consider the case of the unsaturated atmosphere, treating the atmospheric air as a two-component medium characterised by the two thermodynamic variables, viz., potential temperature $\theta$ and specific humidity $q$. The real atmosphere is of course more complicated as it also contains water in liquid and solid forms. Some issues related to modelling turbulence in the cloudy atmosphere are briefly discussed in section 2.5.

The set of governing equations, hereafter referred to as the ensemble-mean equations, consists of the transport equations for the Reynolds stress $\left\langle u_{i}^{\prime} u_{j}^{\prime}\right\rangle$, for the scalar fluxes $\left\langle u_{i}^{\prime} \theta^{\prime}\right\rangle$ and $\left\langle u_{i}^{\prime} q^{\prime}\right\rangle$, for the scalar variances $\left\langle\theta^{\prime 2}\right\rangle$ and $\left\langle q^{\prime 2}\right\rangle$, and for the temperature-humidity covariance $\left\langle\theta^{\prime} q^{\prime}\right\rangle$. Using the Boussinesq approximation and assuming that the Reynolds number is sufficiently high to neglect the molecular diffusion terms in the second-moment budget equations (a good approximation for the majority of atmospheric flows), they read

$$
\begin{gathered}
\left(\frac{\partial}{\partial t}+\left\langle u_{k}\right\rangle \frac{\partial}{\partial x_{k}}\right)\left\langle u_{i}^{\prime} u_{j}^{\prime}\right\rangle=-\left(\left\langle u_{i}^{\prime} u_{k}^{\prime}\right\rangle \frac{\partial\left\langle u_{j}\right\rangle}{\partial x_{k}}+\left\langle u_{j}^{\prime} u_{k}^{\prime}\right\rangle \frac{\partial\left\langle u_{i}\right\rangle}{\partial x_{k}}\right) \\
-\left(\beta_{i}\left\langle u_{j}^{\prime} \theta_{v}^{\prime}\right\rangle+\beta_{j}\left\langle u_{i}^{\prime} \theta_{v}^{\prime}\right\rangle\right)-2\left(\epsilon_{i l k} \Omega_{l}\left\langle u_{k}^{\prime} u_{j}^{\prime}\right\rangle+\epsilon_{j l k} \Omega_{l}\left\langle u_{k}^{\prime} u_{i}^{\prime}\right\rangle\right) \\
-\left(\left\langle u_{i}^{\prime} \frac{\partial p^{\prime}}{\partial x_{j}}\right\rangle+\left\langle u_{j}^{\prime} \frac{\partial p^{\prime}}{\partial x_{i}}\right\rangle-\frac{2}{3} \delta_{i j} \frac{\partial}{\partial x_{k}}\left\langle u_{k}^{\prime} p^{\prime}\right\rangle\right) \\
-\frac{\partial}{\partial x_{k}}\left(\left\langle u_{k}^{\prime} u_{i}^{\prime} u_{j}^{\prime}\right\rangle+\frac{2}{3} \delta_{i j}\left\langle u_{k}^{\prime} p^{\prime}\right\rangle\right)-\epsilon_{i j}, \\
\left(\frac{\partial}{\partial t}+\left\langle u_{k}\right\rangle \frac{\partial}{\partial x_{k}}\right)\left\langle u_{i}^{\prime} \theta^{\prime}\right\rangle=-\left\langle u_{k}^{\prime} \theta^{\prime}\right\rangle \frac{\partial\left\langle u_{i}\right\rangle}{\partial x_{k}}-\left\langle u_{i}^{\prime} u_{k}^{\prime}\right\rangle \frac{\partial\langle\theta\rangle}{\partial x_{k}} \\
-\beta_{i}\left\langle\theta^{\prime} \theta_{v}^{\prime}\right\rangle-2 \epsilon_{i j k} \Omega_{j}\left\langle u_{k}^{\prime} \theta^{\prime}\right\rangle-\left\langle\theta^{\prime} \frac{\partial p^{\prime}}{\partial x_{i}}\right\rangle-\frac{\partial}{\partial x_{k}}\left\langle u_{k}^{\prime} u_{i}^{\prime} \theta^{\prime}\right\rangle-\epsilon_{i \theta}, \\
\frac{\partial}{2}\left(\frac{\partial}{\partial t}+\left\langle u_{k}\right\rangle \frac{\partial}{\partial x_{k}}\right)\left\langle\theta^{\prime 2}\right\rangle=-\left\langle u_{k}^{\prime} \theta^{\prime}\right\rangle \frac{\partial\langle\theta\rangle}{\partial x_{k}}-\frac{1}{2} \frac{\partial}{\partial x_{k}}\left\langle u_{k}^{\prime} \theta^{\prime 2}\right\rangle-\epsilon_{\theta}, \\
-\beta_{i}\left\langle q^{\prime} \theta_{v}^{\prime}\right\rangle-2 \epsilon_{i j k} \Omega_{j}\left\langle u_{k}^{\prime} q^{\prime}\right\rangle-\left\langle q^{\prime} \frac{\partial p^{\prime}}{\partial x_{i}}\right\rangle-\frac{\partial}{\partial x_{k}}\left\langle u_{k}^{\prime} u_{i}^{\prime} q^{\prime}\right\rangle-\epsilon_{i q} \\
\left(u_{i}^{\prime} q^{\prime}\right\rangle=-\left\langle u_{k}^{\prime} q^{\prime}\right\rangle \frac{\partial\left\langle u_{i}\right\rangle}{\partial x_{k}}-\left\langle u_{i}^{\prime} u_{k}^{\prime}\right\rangle \frac{\partial\langle q\rangle}{\partial x_{k}}
\end{gathered}
$$




$$
\begin{gathered}
\frac{1}{2}\left(\frac{\partial}{\partial t}+\left\langle u_{k}\right\rangle \frac{\partial}{\partial x_{k}}\right)\left\langle q^{\prime 2}\right\rangle=-\left\langle u_{k}^{\prime} q^{\prime}\right\rangle \frac{\partial\langle q\rangle}{\partial x_{k}}-\frac{1}{2} \frac{\partial}{\partial x_{k}}\left\langle u_{k}^{\prime} q^{\prime 2}\right\rangle-\epsilon_{q} \\
\left(\frac{\partial}{\partial t}+\left\langle u_{k}\right\rangle \frac{\partial}{\partial x_{k}}\right)\left\langle\theta^{\prime} q^{\prime}\right\rangle=-\left\langle u_{k}^{\prime} \theta^{\prime}\right\rangle \frac{\partial\langle q\rangle}{\partial x_{k}}-\left\langle u_{k}^{\prime} q^{\prime}\right\rangle \frac{\partial\langle\theta\rangle}{\partial x_{k}} \\
-\frac{\partial}{\partial x_{k}}\left\langle u_{k}^{\prime} \theta^{\prime} q^{\prime}\right\rangle-\epsilon_{\theta q} .
\end{gathered}
$$

Here, $\theta_{v}=\theta\left[1+\left(R_{v} / R_{d}-1\right) q\right] \approx \theta(1+0.608 q)$ is the virtual potential temperature, $R_{v}$ and $R_{d}$ are the gas constants for water vapour and for dry air, respectively, $\beta_{i}=g_{i} / \theta_{r}$ is the buoyancy parameter, $g_{i}$ is the acceleration due to gravity, $\theta_{r}$ is the reference value of temperature, $\Omega_{i}$ is the angular velocity of the earth's rotation, and $p$ is the kinematic pressure (deviation of pressure from the hydrostatically balanced pressure divided by the reference density $\left.\rho_{r}\right)$. The dissipation rates of various quantities are denoted by $\epsilon_{i j}, \epsilon_{i \theta}, \epsilon_{i q}, \epsilon_{\theta}$, $\epsilon_{q}$, and $\epsilon_{\theta q}$.

Taking the trace of Eq. (3) yields the budget equation for the TKE $e=$ $\frac{1}{2}\left\langle u_{i}^{\prime 2}\right\rangle$,

$$
\begin{gathered}
\frac{1}{2}\left(\frac{\partial}{\partial t}+\left\langle u_{k}\right\rangle \frac{\partial}{\partial x_{k}}\right)\left\langle u_{i}^{\prime 2}\right\rangle=-\left\langle u_{i}^{\prime} u_{k}^{\prime}\right\rangle \frac{\partial\left\langle u_{i}\right\rangle}{\partial x_{k}}-\beta_{i}\left\langle u_{i}^{\prime} \theta_{v}^{\prime}\right\rangle \\
-\frac{\partial}{\partial x_{k}}\left(\frac{1}{2}\left\langle u_{k}^{\prime} u_{i}^{\prime 2}\right\rangle+\left\langle u_{k}^{\prime} p^{\prime}\right\rangle\right)-\epsilon
\end{gathered}
$$

where $\epsilon=\frac{1}{2} \epsilon_{i i}$ is the TKE dissipation rate.

It should be noted that the decomposition of the pressure gradient-velocity correlation $\left(\left\langle u_{j}^{\prime} \partial p^{\prime} / \partial x_{i}\right\rangle+\left\langle u_{i}^{\prime} \partial p^{\prime} / \partial x_{j}\right\rangle\right)$ that appears in the Reynolds stress equation (3) is not unique. Along with a more traditional decomposition into pressure-strain and pressure-diffusion, the decomposition into deviatoric and isotropic parts has also been advocated (e.g. [185]). Keeping in mind that the issue is not resolved, the former decomposition is adopted here.

\subsection{Closure Assumptions}

The second-order equations (3)-(9) are not closed as they contain a number of unknown quantities. There are three groups of unknowns, namely, the pressure-velocity and pressure-scalar covariances, the third-order velocityvelocity and velocity-scalar covariances, and the dissipation rates of the second-order moments. Parameterisations (closure assumptions) are required for these quantities to express them in terms of the first-order and the second-order moments involved and thereby close the system of governing equations. A large number of parameterisations have been developed to 
date that vary greatly in terms of their complexity and field of application. These parameterisations are the subject of an extremely voluminous literature. General scope reviews are given in e.g. [195, 131, 85, 121, 161]. Reviews of second-order closures for geophysical applications are given in [143, 155, 170, 99, 37, 61, 203, 16, 186], among others. In this section, we consider parameterisations typically utilised in the second-order modelling of geophysical flows. We briefly discuss their advantages and limitations, emphasising their utility for modelling turbulence in the lower troposphere.

One remark is in order. Plausible parameterisations used in the secondorder equations should satisfy a number of physical and mathematical requirements. Apart from the requirements of proper physical dimensions, tensor invariance and symmetry, the so-called realisability requirements should also be met. The concept of realisability $[171,57,131]$ states that the Schwarz' inequalities for all turbulence moments must always be satisfied. For the secondorder moments, this means that the velocity variances and the scalar variances must always be non-negative and that the magnitude of the correlation coefficient $\left\langle a^{\prime} b^{\prime}\right\rangle /\left(\left\langle a^{\prime 2}\right\rangle\left\langle b^{\prime 2}\right\rangle\right)^{1 / 2}$ between any two fluctuating quantities $a$ and $b$ must not exceed 1 . Intrinsically realisable models do not generate, by virtue of their construction, physically impossible results. Notice that the realisability constraints not only help to develop turbulence models that possess desired physical and mathematical properties. They are also useful in that they provide additional relations between the model coefficients, thus reducing the number of undetermined coefficients to be evaluated (tuned) on the basis of empirical and/or numerical data.

\section{Pressure Terms}

Rotta [169] proposed a return-to-isotropy parameterisation for the pressurevelocity gradient covariance in turbulent shear flows. That parameterisation states that the rate of return of turbulence to isotropy is proportional to the degree of anisotropy and inversely proportional to a certain time scale called "return-to-isotropy" time scale. Then, the pressure redistribution term, the fourth term on the r.h.s. of Eq. (3) that we denote by $\Pi_{i j}$, is given by

$$
\begin{gathered}
\left(\left\langle u_{i}^{\prime} \frac{\partial p^{\prime}}{\partial x_{j}}\right\rangle+\left\langle u_{j}^{\prime} \frac{\partial p^{\prime}}{\partial x_{i}}\right\rangle-\frac{2}{3} \delta_{i j} \frac{\partial}{\partial x_{k}}\left\langle u_{k}^{\prime} p^{\prime}\right\rangle\right) \equiv \\
\Pi_{i j}=\frac{\left\langle u_{i}^{\prime} u_{j}^{\prime}\right\rangle-\frac{1}{3} \delta_{i j}\left\langle u_{k}^{\prime} u_{k}^{\prime}\right\rangle}{\tau_{u *}},
\end{gathered}
$$

where $\tau_{u *}$ is the return-to-isotropy time scale. The return-to-isotropy formulation was extended to the pressure gradient-scalar covariance, assuming that the rate of destruction of turbulent scalar flux is related to the flux in question through a certain relaxation time scale. By way of example, consider the 
formulation for the potential temperature. Other scalars can be treated in much the same way. Denoting the pressure gradient-temperature covariance, the fifth term on the r.h.s. of Eq. (4), by $\Pi_{\theta i}$, we get

$$
\left\langle\theta^{\prime} \frac{\partial p^{\prime}}{\partial x_{i}}\right\rangle \equiv \Pi_{\theta i}=\frac{\left\langle u_{i}^{\prime} \theta^{\prime}\right\rangle}{\tau_{\theta *}},
$$

where $\tau_{\theta *}$ is the relaxation "return-to-isotropy" time scale for potential temperature.

Applying return-to-isotropy parameterisations to the entire pressure terms, one lumps all the uncertainties on the relaxation time scales. A more common approach nowadays is to decompose $\Pi_{i j}$ and $\Pi_{\theta i}$ into the contributions due to the non-linear turbulence interactions, mean shear, buoyancy, and the Coriolis effects, and to model these contributions separately. Then, the return-toisotropy parameterisation is applied to the non-linear turbulence contributions only. The approximations for the pressure terms $\Pi_{i j}$ and $\Pi_{\theta i}$ can be written in the form (see e.g. [215, 145, 203]):

$$
\begin{gathered}
\Pi_{i j}=C_{t}^{u} \frac{a_{i j}}{\tau_{u}} e \\
+\left[C_{s 1}^{u} S_{i j}+C_{s 2}^{u}\left(a_{i k} S_{k j}+a_{j k} S_{k i}-\frac{2}{3} \delta_{i j} a_{k l} S_{k l}\right)+C_{s 3}^{u}\left(a_{i k} W_{k j}+a_{j k} W_{k i}\right)\right] e \\
+C_{b}^{u}\left(\beta_{i}\left\langle u_{j}^{\prime} \theta_{v}^{\prime}\right\rangle+\beta_{j}\left\langle u_{i}^{\prime} \theta_{v}^{\prime}\right\rangle-\frac{2}{3} \delta_{i j} \beta_{k}\left\langle u_{k}^{\prime} \theta_{v}^{\prime}\right\rangle\right) \\
+2 C_{c}^{u}\left(\epsilon_{i l k} \Omega_{l}\left\langle u_{k}^{\prime} u_{j}^{\prime}\right\rangle+\epsilon_{j l k} \Omega_{l}\left\langle u_{k}^{\prime} u_{i}^{\prime}\right\rangle\right)+N L T \\
\Pi_{\theta i}=C_{t}^{\theta} \frac{\left\langle u_{i}^{\prime} \theta^{\prime}\right\rangle}{\tau_{\theta}}+\left(C_{s 1}^{\theta} S_{i j}+C_{s 2}^{\theta} W_{i j}\right)\left\langle u_{j}^{\prime} \theta^{\prime}\right\rangle \\
+C_{b}^{\theta} \beta_{i}\left\langle\theta^{\prime} \theta_{v}^{\prime}\right\rangle+2 C_{c}^{\theta} \epsilon_{i j k} \Omega_{j}\left\langle u_{k}^{\prime} \theta^{\prime}\right\rangle+N L T .
\end{gathered}
$$

Here, $a_{i j}=2 \frac{\left\langle u_{i}^{\prime} u_{j}^{\prime}\right\rangle}{\left\langle u_{k}^{\prime} u_{k}^{\prime}\right\rangle}-\frac{2}{3} \delta_{i j}$ is the departure-from-isotropy tensor, $S_{i j}=$ $\frac{1}{2}\left(\frac{\partial\left\langle u_{i}\right\rangle}{\partial x_{j}}+\frac{\partial\left\langle u_{j}\right\rangle}{\partial x_{i}}\right)$ and $W_{i j}=\frac{1}{2}\left(\frac{\partial\left\langle u_{i}\right\rangle}{\partial x_{j}}-\frac{\partial\left\langle u_{j}\right\rangle}{\partial x_{i}}\right)$ are the symmetric and the antisymmetric parts of the mean-velocity gradient tensor, respectively, and $C_{t}^{u}, C_{s 1}^{u}, C_{s 2}^{u}, C_{s 3}^{u}, C_{b}^{u}, C_{c}^{u}, C_{t}^{\theta}, C_{s 1}^{\theta}, C_{s 2}^{\theta}, C_{b}^{\theta}$ and $C_{c}^{\theta}$ are dimensionless coefficients. The return-to-isotropy time scales $\tau_{u}$ and $\tau_{\theta}$ should not be confused with $\tau_{u *}$ and $\tau_{\theta *}$ in Eqs. (10) and (11).

Jones and Musogne [94] (see also [50]) added an additional term to the parameterisation of $\Pi_{\theta i}$ that is proportional to the gradient of mean scalar 
concentration. It reads $C_{m}^{\theta} e a_{i j} \partial\langle\theta\rangle / \partial x_{j}$, where $C_{m}^{\theta}$ is a dimensionless coefficient. The Jones and Musogne approach was further developed by Craft et al. [46] who incorporated the mean-gradient term into the expression for the non-linear turbulence (return-to-isotropy) contribution to $\Pi_{\theta i}$.

The mean-shear, buoyancy and Coriolis terms on the r.h.s. of Eqs. (12) and (13) represent linear contributions to the so-called rapid parts of $\Pi_{i j}$ and $\Pi_{\theta i}$ (the return-to-isotropy contributions to $\Pi_{i j}$ and $\Pi_{\theta i}$ are referred to as slow parts of the pressure terms). Notice that these linear rapid terms have the same form as the mean-shear, buoyancy and Coriolis terms in Eq. (3) for the Reynolds stress and Eq. (4) for the temperature flux. Therefore, the effect of linear rapid terms, sometimes referred to as the implicit mean-shear, buoyancy and Coriolis terms, is simply to partially offset the respective explicit terms already present in Eqs. (3) and (4).

Generally speaking, the pressure-velocity and the pressure-scalar covariances depend non-linearly on the departure-from-isotropy tensor and on the other tensors involved, e.g. on the rotation tensor $\epsilon_{i k j} \Omega_{k}$. The non-linear parts of $\Pi_{i j}$ and $\Pi_{\theta i}$ are symbolised by NLT on the r.h.s. of Eqs. (12) and (13). Numerous elaborate non-linear formulations of the pressure terms have been proposed (e.g. [131, 215, 167, 46, 85, 145]). Particular emphasis is placed on the realisability of parameterised pressure terms, following different ways of imposing realisability constraints. The non-linear models perform better than the simplified linear models, particularly in flows with large departures from isotropy. However, the non-linear models are inevitably complex. They are often inconvenient to use and are computationally expensive. It is therefore common practice in geophysical applications to put up with the shortcomings of linear models and apply Eqs. (12) and (13) without NLTs. In doing so the TKE dissipation time scale $\tau_{\epsilon}=e / \epsilon$ is typically used instead of the return-to-isotropy time scales $\tau_{u}$ and $\tau_{\theta}$, assuming that all these time scales are proportional to each other. The dimensionless coefficients $C_{t}^{u}$ through $C_{c}^{\theta}$ in Eqs. (12) and (13) are adjusted to provide a good fit of the model results to empirical data, i.e. these coefficients are treated as tuning model parameters. Some estimates of these dimensionless coefficients used in geophysical turbulence modelling are given in [203]. Several important points should be discussed in relation to the parameterisation of the pressure terms.

First and foremost we recall how the parameterisations for the pressure terms are derived. Taking the divergence of the transport equation for the fluctuating velocity, a Poisson equation for the fluctuating pressure is obtained. Parameterisations for various contributions to $\Pi_{i j}$ and $\Pi_{\theta i}$ are then developed on the basis of the Green's function solution to the Poisson equation [45]. That solution depends on the entire fluid domain considered. In practice, however, the two-point correlations are assumed to be different from zero only in the vicinity of the point where the pressure terms are evaluated. Then, the pressure-velocity and pressure-scalar covariances are modelled as if they were local (dependent on the flow variables at the same point), although they may actually be non-local (dependent on the flow variables in the entire domain). 
Therefore, formulations of the type given by Eqs. (12) and (13) have inherent limitations. These formulations may not perform well in situation where turbulence is essentially non-local, as is, for example, the case for atmospheric convection. Notice that both linear and non-linear one-point formulations for $\Pi_{i j}$ and $\Pi_{\theta i}$ suffer from this shortcoming.

Although linear models of $\Pi_{i j}$ and $\Pi_{\theta i}$ are attractive from the standpoint of practical applications, they may entirely fail in some situations of interest. An illustrative example is turbulent convection driven by the surface buoyancy flux and affected by rotation $[148,145]$. In the seemingly simple case where the rotation axis is aligned with the vector of gravity, the linear model predicts a Coriolis contribution to $\Pi_{\theta i}$ that is identically zero, although the Coriolis contribution becomes one of the dominant parts of $\Pi_{\theta i}$ as the rotation rate increases. As shown in [145], a non-linear formulation is required which is at least quadratic in the rotation tensor $\epsilon_{i k j} \Omega_{k}$. Fortunately, the effect of rotation on turbulence is of little importance in most atmospheric flows. ${ }^{2}$ Similar problems may, however, be encountered when the effects of buoyancy and shear are considered. Caution must be exercised when simplified parameterisations are applied.

To conclude this section, we remark that modelling the pressure transport term $\frac{2}{3} \delta_{i j} \partial\left\langle u_{k}^{\prime} p^{\prime}\right\rangle / \partial x_{k}$ in Eq. (3) represents a separate problem. Lumley [131] and Shih [174] discussed this problem in some detail. The pressure transport term is usually smaller than the third-order velocity correlation term, although this is not always the case. It is standard practice in applied turbulence modelling to neglect the pressure transport term entirely, or to incorporate it into a parameterisation of the third-order velocity correlation.

\section{Third-Order Moments}

The second group of terms that require closure assumptions includes turbulence moments of the third-order. These terms enter Eqs. (3)-(9) in the divergence form. They describe the transport of the second-order moments by the fluctuating velocity. Numerous formulations have been proposed for the third-order transport terms (e.g. [49, 86, 118, 43, 83]), ranging from the simplest down-gradient approximations to very complex formulations based on a sophisticated treatment of transport equations for the third-order turbulence moments. Simple down-gradient approximations have been most popular in geophysical applications. They read

$$
\left\langle u_{i}^{\prime} u_{j}^{\prime} u_{k}^{\prime}\right\rangle=-K_{u u}\left(\frac{\partial\left\langle u_{i}^{\prime} u_{j}^{\prime}\right\rangle}{\partial x_{k}}+\frac{\partial\left\langle u_{i}^{\prime} u_{k}^{\prime}\right\rangle}{\partial x_{j}}+\frac{\partial\left\langle u_{j}^{\prime} u_{k}^{\prime}\right\rangle}{\partial x_{i}}\right),
$$

${ }^{2}$ This holds for deep convective updraughts, boundary-layer plumes and rolls, and eddy motions on a smaller scale. Eddy motions of larger spatial scale, such as synoptic weather systems (e.g. cyclones and fronts) and regional circulations, do feel the earth's rotation. These motions are, however, resolved by the present-day NWP models so that there is no need for a parameterisation. 


$$
\begin{gathered}
\left\langle u_{i}^{\prime} u_{j}^{\prime} \theta^{\prime}\right\rangle=-K_{u \theta}\left(\frac{\partial\left\langle u_{i}^{\prime} \theta^{\prime}\right\rangle}{\partial x_{j}}+\frac{\partial\left\langle u_{j}^{\prime} \theta^{\prime}\right\rangle}{\partial x_{i}}\right), \\
\left\langle u_{i}^{\prime} \theta^{\prime 2}\right\rangle=-K_{\theta \theta} \frac{\partial\left\langle\theta^{\prime 2}\right\rangle}{\partial x_{i}},
\end{gathered}
$$

where $K_{u u}, K_{u \theta}$ and $K_{\theta \theta}$ are the eddy diffusion coefficients. Other scalar quantities are treated in the same way as the potential temperature. The down-gradient approximation for the TKE transport term reads

$$
\left\langle u_{i}^{\prime} u_{k}^{\prime 2}\right\rangle=-K_{e} \frac{\partial\left\langle u_{k}^{\prime 2}\right\rangle}{\partial x_{i}},
$$

where $K_{e}$ is the eddy diffusion coefficient with respect to the TKE.

The down-gradient formulations (14)-(17) are attractive for their simplicity. It has long since been recognised, however, that their performance in complex flows leaves very much to be desired and a more accurate treatment of the third-order moments is required. This is particularly true for convective flows (e.g. [151, 148]), but may also be the case for stably stratified flows (e.g. [46]). In an attempt to develop a physically plausible parameterisation, the focus has been on buoyant convection where the third-order moments are largely responsible for non-local transport properties of turbulent motions.

A straightforward way is to derive expressions for the third-order moments from their budget equations. These equations require closure assumptions in much the same way as the second-moment equations. In particular, the fourthorder moments that describe the transport of the third-order quantities by the fluctuating velocity should be parameterised. The so-called Millionshchikov hypothesis [144] has been used for this purpose over several decades. It states that the fourth-order moments can be considered as quasi-Gaussian, even though the third-order moments are non-zero. That is, the following relation holds for any four fluctuating quantities $a, b, c$ and $d$ :

$$
\left\langle a^{\prime} b^{\prime} c^{\prime} d^{\prime}\right\rangle=\left\langle a^{\prime} b^{\prime}\right\rangle\left\langle c^{\prime} d^{\prime}\right\rangle+\left\langle a^{\prime} c^{\prime}\right\rangle\left\langle b^{\prime} d^{\prime}\right\rangle+\left\langle a^{\prime} d^{\prime}\right\rangle\left\langle b^{\prime} c^{\prime}\right\rangle .
$$

Using Eq. (18) for the fourth-order moments, the Rotta-type formulations for the pressure terms, and the relaxation-type formulations for the dissipation terms, then neglecting the advection and the time-rate-of-change of the third-order moments, a closed set of algebraic expressions for the third-order moments is derived. Canuto et al. [43] developed such expressions for the horizontally-homogeneous convective boundary layer (CBL). The third-order moments appear to be linear combinations of the derivatives (in the $x_{3}$ vertical direction only) of all second-order moments involved multiplied by certain combinations of governing parameters with dimensions of eddy diffusivity. Some of those combinations explicitly depend on the buoyancy parameter. Canuto et al. [42] employed a modified quasi-normal approximation that basically amounts to multiplying the r.h.s. of Eq. (18) by a correction function 
of the dissipation time scale and of the buoyancy time scale (a reciprocal of the buoyancy frequency). These authors proposed modified (and slightly simplified) expressions for the third-order moments that show a better agreement with large-eddy simulation (LES) data from a shear-free CBL than the expressions given in [43].

An attractive way of looking at the problem of non-local convective transport is based on the observation that convective turbulence is skewed. For example, in the CBL driven by the surface buoyancy flux, the vertical transport in mid-CBL is dominated by quasi-organised motions, convective updraughts, whose size is of the order of the CBL depth. The updraughts are more localised (occupy a smaller area) than the compensating downward motions, downdraughts. A quantitative measure of this localisation is the verticalvelocity skewness $S_{w}=\left\langle u_{3}^{\prime 3}\right\rangle /\left\langle u_{3}^{\prime 2}\right\rangle^{3 / 2}$. Likewise the potential-temperature skewness $S_{\theta}=\left\langle\theta^{\prime 3}\right\rangle /\left\langle\theta^{\prime 2}\right\rangle^{3 / 2}$ is a quantitative measure of the localisation of potential-temperature anomalies (with respect to a horizontal mean). Guided by this view of convective circulation and of the bottom-up top-down transport asymmetry [210], Abdella and McFarlane [1], Canuto and Dubovikov [41], and Zilitinkevich et al. [227] proposed the following parameterisation for the flux of potential-temperature flux:

$$
\left\langle u_{3}^{\prime 2} \theta^{\prime}\right\rangle=S_{w}\left\langle u_{3}^{\prime 2}\right\rangle^{1 / 2}\left\langle u_{3}^{\prime} \theta^{\prime}\right\rangle .
$$

This expression has an advective rather than a down-gradient diffusive form, indicating that the temperature flux is transported by the CBL-scale quasiorganised eddies rather than diffused by small-scale random turbulence. The quantity $S_{w}\left\langle u_{3}^{\prime 2}\right\rangle^{1 / 2}=\left\langle u_{3}^{\prime 3}\right\rangle /\left\langle u_{3}^{\prime 2}\right\rangle$ was termed "large-eddy skewedturbulence advection velocity" in [227]. Zilitinkevich et al. [227] (see also [83]) added a conventional down-gradient diffusion term $-K_{w \theta} \partial\left\langle u_{3}^{\prime} \theta^{\prime}\right\rangle / \partial x_{3}, K_{w \theta}$ being the turbulent diffusivity with respect to $\left\langle u_{3}^{\prime} \theta^{\prime}\right\rangle$, to the r.h.s. of Eq. (19) in order to arrive at an interpolation formula that should work in both wellmixed regions of the flow, where advective transport by the CBL-scale eddies dominates, and in stratified regions, where turbulent transport is primarily of diffusive character.

A skewness-dependent parameterisation for the flux of potential-temperature variance was formulated by Mironov et al. [147], Abdella and McFarlane [2], and Abdella and Petersen [3]. It reads

$$
\left\langle u_{3}^{\prime} \theta^{\prime 2}\right\rangle=S_{\theta}\left\langle\theta^{\prime 2}\right\rangle^{1 / 2}\left\langle u_{3}^{\prime} \theta^{\prime}\right\rangle .
$$

An interpolation formula for $\left\langle u_{3}^{\prime} \theta^{\prime 2}\right\rangle$ that incorporates the down-gradient term $-K_{\theta \theta} \partial\left\langle\theta^{\prime 2}\right\rangle / \partial x_{3}, K_{\theta \theta}$ being the turbulent diffusivity with respect to $\left\langle\theta^{\prime 2}\right\rangle$, was presented in [83].

Equations (19) and (20) are consistent with the top-hat representation of fluctuating quantities. The top-hat representation is central to the mass-flux approach widely used to parameterise convection in numerical models of the 
atmosphere. The simplest top-hat mass-flux model can be formulated in terms of a probability distribution function (PDF) which consists of only two Dirac delta functions, i.e. the probabilities of motions to be either updraughts or downdraughts are $P_{u}$ and $P_{d}$, respectively, and $P_{u}+P_{d}=1$. A comprehensive account of the two-delta-function mass-flux framework is given by Randall et al. [163], Lappen and Randall [111, 112], and Gryanik and Hartmann [83]. In order to emphasise a different localisation (different fractional area coverage of positive/negative anomalies with respect to a horizontal mean) of the vertical velocity and of the scalar quantities, as manifested by a difference between $S_{w}$ and $S_{\theta}$ (see Fig. 1 in [147]), Gryanik and Hartmann [83] refer to their approach as to the two-scale mass-flux approach. Notice that different PDFs can be used to develop parameterisations of statistical moments of turbulence. For example, Larson and Golaz [117] developed parameterisations of various third-order and fourth-order moments, using a combination of two trivariate Gaussian functions. These authors considered moist CBL and presented their results in terms of vertical velocity, liquid water potential temperature and total water specific humidity. The formulations based on the two-Gaussianfunction PDF revealed a somewhat improved fit to data for some moments as compared to the formulations based on the two-delta-function PDF.

Equations (19) and (20) require that $S_{w}$ and $S_{\theta}$ be specified. If the budget equations for the third-order moments are used for this purpose as discussed above, formulations for the fourth-order moments are required. Taking the two-scale mass-flux approach, Gryanik and Hartmann [83] and Gryanik et al. [84] proposed (see also [2])

$$
\begin{gathered}
\left\langle u_{3}^{\prime 4}\right\rangle=3\left(1+\frac{1}{3} S_{w}^{2}\right)\left\langle u_{3}^{\prime 2}\right\rangle^{2}, \quad\left\langle\theta^{\prime 4}\right\rangle=3\left(1+\frac{1}{3} S_{\theta}^{2}\right)\left\langle\theta^{\prime 2}\right\rangle^{2} \\
\left\langle u_{3}^{\prime 3} \theta^{\prime}\right\rangle=3\left(1+\frac{1}{3} S_{w}^{2}\right)\left\langle u_{3}^{\prime 2}\right\rangle\left\langle u_{3}^{\prime} \theta^{\prime}\right\rangle \\
\left\langle u_{3}^{\prime} \theta^{\prime 3}\right\rangle=3\left(1+\frac{1}{3} S_{\theta}^{2}\right)\left\langle\theta^{\prime 2}\right\rangle\left\langle u_{3}^{\prime} \theta^{\prime}\right\rangle, \\
\left\langle u_{3}^{\prime 2} \theta^{\prime 2}\right\rangle=\left\langle u_{3}^{\prime 2}\right\rangle\left\langle\theta^{\prime 2}\right\rangle+2\left\langle u_{3}^{\prime} \theta^{\prime}\right\rangle^{2}+S_{w} S_{\theta}\left\langle u_{3}^{\prime} \theta^{\prime}\right\rangle\left\langle u_{3}^{\prime 2}\right\rangle^{1 / 2}\left\langle\theta^{\prime 2}\right\rangle^{1 / 2}
\end{gathered}
$$

Similar expressions for the fourth-order moments that incorporate horizontal velocity components $u_{1}$ and $u_{2}$ are presented in [84]. Both Eqs. (19) and (20) for the third-order moments and Eqs. (21)-(24) for the fourth-order moments were favourably tested against data from LES and from aircraft measurements in the atmospheric CBL $[83,84]$, from numerical simulation of open-ocean deep convection [129], and from numerical simulation of solar and stellar convection [109]. 
Equations (21)-(24) amount to a generalisation of the Millionshchikov hypothesis. Indeed, in the case of isotropic turbulence, $S_{w}$ and $S_{\theta}$ vanish and Eqs. (21)-(24) reduce to the form given by Eq. (18). In the other limiting case of very skewed turbulence, the terms with $S_{w}$ and $S_{\theta}$ dominate over the other terms and Eqs. (21)-(24) take on the form suggested by the top-hat mass-flux approach. Then, Eqs. (21)-(24) represent the simplest linear interpolation between the two limiting cases, where dimensionless coefficients on the r.h.s. $(3,1$ and $1 / 3)$ are chosen in such a way that these limiting cases are satisfied exactly. It should be emphasised that Eqs. (19) and (20) for the thirdorder moments and Eqs. (21)-(24) for the fourth-order moments taken in the limit of large skewness are in essence the top-hat mass-flux parameterisations expressed in terms of the ensemble-mean quantities. Analogies between the ensemble-mean and the mass-flux modelling frameworks are discussed below in greater depth.

Noteworthy also is that the expressions (21)-(24) for the fourth-order moments satisfy the realisability constraints $[5,6,7,84]$ regardless of the magnitude of skewness. This is not the case for Eq. (18) that violates realisability if the magnitude of $S_{w}$ or of $S_{\theta}$ exceeds $2^{1 / 2}$ [84].

Parameterisations (19)-(24) are developed for the temperature-stratified horizontally-homogeneous CBL, where potential temperature is the only thermodynamic variable and all quantities of interest depend on the $x_{3}$ vertical co-ordinate only. Their extension to the three-dimensional case is by no means trivial but seems to be manageable (D. Mironov, A note on the parameterisation of the third-order transport in skewed convective boundary-layer turbulence, unpublished manuscript; V. Gryanik, personal communication). Such an extension is highly desirable and should be developed. The same is true for the extension to the moist atmosphere, where, apart from potential temperature, water in its three phases should be considered.

\section{Dissipation Rates}

Finally, the rates of dissipation of the second-order turbulence moments should be parameterised. It is common practice to assume, following Kolmogorov [105], local isotropy at small scales, giving $\epsilon_{i \theta}=0, \epsilon_{i q}=0$, and $\epsilon_{i j}=\frac{2}{3} \delta_{i j} \epsilon$. In order to determine the TKE dissipation rate, a prognostic equation for $\epsilon$ has been used in engineering and geophysics over several decades (e.g. $[49,118,168,58,10,46,37,202,16])$. The dissipation rates of the scalar quantities, $\epsilon_{\theta}, \epsilon_{q}$ and $\epsilon_{\theta q}$, are either related to $e$ and $\epsilon$ through $\epsilon_{\theta} \propto e^{-1} \epsilon\left\langle\theta^{\prime 2}\right\rangle$ (similarly for $\epsilon_{q}$ and $\epsilon_{\theta q}$ ), or computed from their own prognostic equations (e.g. [157]). Once the dissipation rates are determined, the various time scales, length scales and eddy diffusion coefficients are computed diagnostically through these dissipation rates and the corresponding variances. For example, the quantities with respect to the TKE are given by $l \propto \epsilon^{-1} e^{3 / 2}$, $\tau \propto \epsilon^{-1} e$ and $K \propto \epsilon^{-1} e^{2}$. In this way the system of the second-order equations is closed. 
Apart from the $\epsilon$ equation, prognostic equations have been formulated for other quantities that determine the turbulence length/time scale. Prognostic equations for the product $e l$ of the TKE and the turbulence length scale [143], for the reciprocal of turbulence time scale $e^{-1} \epsilon$ [206, 204], and for the eddy diffusivity $\epsilon^{-1} e^{2}$ [214] are examples. These closure ideas were generalised by Umlauf and Burchard [202] who developed a generic equation for the quantity $e^{m} l^{n}$ that incorporates the equations mentioned above as particular cases. These authors proposed a rational way to calibrate their generalised model in the so-called two-equation second-order modelling framework, where only the TKE equation and the equation for $e^{m} l^{n}$ are carried as prognostic equations whereas the other second-order equations are reduced to the diagnostic algebraic expressions. The exponents $m$ and $n$ along with the other model parameters are evaluated by demanding consistency with a number of welldocumented reference cases, such as the logarithmic boundary layer and the decay of homogeneous turbulence.

The prognostic equations for the dissipation rates of TKE and of scalar variances are very complex. They contain a number of terms whose physical nature is not satisfactorily understood. In fact, all terms in the dissipationrate equations that describe production, destruction and turbulent transport of the dissipation rates should be parameterised, and the validity of those parameterisations is uncertain. It has often been questioned whether prognostic equations for the dissipation rates are really necessary, or diagnostic expressions may be sufficient, at least in case of a relatively simple flow geometry. The latter viewpoint is often held in geophysical applications. A simple and an economical way to determine the dissipation rates of the TKE and of the scalar variances is to compute them from the following expressions:

$$
\epsilon=C_{\epsilon e} \frac{e^{3 / 2}}{l}, \quad \epsilon_{\theta}=C_{\epsilon \theta} \frac{\left\langle\theta^{\prime 2}\right\rangle e^{1 / 2}}{l},
$$

using one or the other formulation for the turbulence length scale $l$. Here, $C_{\epsilon e}$ and $C_{\epsilon \theta}$ are dimensionless coefficients. The dissipation rates $\epsilon_{q}$ and $\epsilon_{\theta q}$ of the humidity variance and of the potential temperature-humidity covariance, respectively, are computed similarly to $\epsilon_{\theta}$. The above expressions for the dissipation rates can be recast in terms of the turbulence time scale $\tau=e^{-1 / 2} l$.

There have been numerous proposals for expressions to compute the length scale $l$. The simplest of them seems to have been Blackadar's formula [28], $l^{-1}=l_{\mathrm{sfc}}^{-1}+l_{\infty}^{-1}$, that interpolates between the two limits, namely, $l=l_{\mathrm{sfc}}=$ $\kappa x_{3}, \kappa$ being the von Kármán constant, as $x_{3} \rightarrow 0$, and $l=l_{\infty}$ as $x_{3} \rightarrow \infty$. This yields the logarithmic profiles close to the underlying surface and prevents the turbulence length scale from growing without bound well above the surface. The free-flow length scale $l_{\infty}$ is either set proportional to the PBL depth, or simply set to a constant value (typically from one hundred to a few hundred metres in the atmospheric models). One more formulation is due to Mellor and Yamada [142] who proposed $l_{\infty}=C_{\infty}\left(\int_{0}^{\infty} x_{3} e^{1 / 2} d x_{3}\right)^{-1} \int_{0}^{\infty} e^{1 / 2} d x_{3}$, where $C_{\infty}$ is a dimensionless coefficient of order $10^{-1}$. Other limitations on $l$ have 
also been used. The length scale is taken to be limited by the shear length scale $l_{s}=C_{l s}\left(S_{i j} S_{i j}\right)^{-1 / 2} e^{1 / 2}$ (e.g. [44] and references therein), and, in case of stable density stratification, by the buoyancy length scale $l_{b}=C_{l b} N^{-1} e^{1 / 2}$, where $N$ is the buoyancy frequency (e.g. [217, 34, 228]). In rotating flows, the length scale is taken to be limited by $l_{r}=C_{l r}\left(\Omega_{i} \Omega_{i}\right)^{-1 / 2} e^{1 / 2}$ (e.g. [87]). This limitation is of little importance in the atmosphere. It is important in many geophysical, astrophysical and technical applications. A prominent geophysical example is open-ocean deep convection [138]. Dimensionless constants $C_{l s}, C_{l b}$ and $C_{l r}$ are evaluated on the basis of empirical and numerical data. Readers are referred to chapter 4 of this volume for further discussion of the dissipation rates and of the turbulence length scale.

\subsection{Simplifications}

The second-order equations closed as discussed above would probably do a fairly nice job of describing most salient features of turbulence in the lower troposphere. However, the full set of the (time-dependent, three-dimensional) second-order equations is still far too complex and expensive computationally. Further simplifications are necessary in order to obtain a reasonably simple turbulence parameterisation scheme that can be accommodated by a numerical model of the atmosphere ${ }^{3}$.

\section{Truncation}

A family of second-order closures has been developed by Mellor and Yamada [142] (see also [143], a comprehensive discussion of the Mellor and Yamada closures and their numerous derivatives is given in [155]). They utilised the second invariant $A_{2}=a_{i j} a_{i j}$ of the departure-from-isotropy tensor as the scaling parameter that measures the degree of flow anisotropy. Using the observation that $A_{2}$ is small and invoking additional arguments to scale the advection and the turbulent diffusion terms, they successively discarded terms of different order in $A_{2}$ in the second-moment equations. The result proved to be a hierarchy of truncated turbulence closure schemes, ranging from the complete second-order closure to a simple algebraic stress model, where all second-moment equations are reduced to algebraic expressions. That hierarchy of closure schemes has found a wide utility in geophysical applications and is often referred to collectively as the Mellor-Yamada closures ever since.

The scheme termed the level 2.5 Mellor-Yamada scheme has been most popular in practical applications. The only prognostic equation carried by that scheme is the TKE equation. The TKE diffusion is usually parameterised through the simplest down-gradient approximation. All other second-moment

${ }^{3}$ The material in sections $2.3,2.4$ and 2.5 is somewhat more technical. Some readers may prefer to proceed directly to section 3 for an outline of the mass-flux convection schemes. 
equations are reduced to algebraic expressions by neglecting the time-rate-ofchange, the advection and the turbulent diffusion terms. The pressure-velocity and the pressure-scalar covariances are parameterised through Eqs. (12) and (13), typically without non-linear terms, or simply through the Rotta-type return-to-isotropy formulations (10) and (11). Notice that the use of Eqs. (12) and (13) without non-linear terms instead of Eqs. (10) and (11) does not radically change the result. Since the linear rapid terms on the r.h.s. of Eqs. (12) and (13) have the same form as the respective terms in Eqs. (3) and (4), the only (though not unimportant) effect of their inclusion is to modify dimensionless coefficients in front of various terms in the resulting expressions for the Reynolds stress and for the scalar fluxes. The dissipation rates of the TKE and of the scalar variances are parameterised through the algebraic relations (25). A turbulence model that carries only one prognostic equation, namely, the TKE equation, is referred to as the one-equation model. In case the transport equation is used for the TKE dissipation rate, or for any quantity $e^{m} l^{n}$ (see above), the resulting turbulence model is referred to as the two-equation model.

\section{Boundary-Layer Approximation}

Another simplification typically involved in geophysical applications is the socalled boundary-layer approximation where the flow is treated as horizontallyhomogeneous. This approximation is fairly accurate for large-scale and mesoscale NWP models, whose grid-box aspect ratio (the ratio of the horizontal grid size to the vertical grid size) is large. In the framework of the boundarylayer approximation, all derivatives in $x_{1}$ and $x_{2}$ horizontal directions in the second-moment equations are neglected and the grid-box mean vertical velocity $\left\langle u_{3}\right\rangle$ is set to zero (in the second-moment equations, but not in the equations for the mean fields). The one-equation turbulence closure scheme in the boundary-layer approximation has been probably the most popular turbulence scheme in NWP. The scheme carries the prognostic TKE equation. All other second-moment equations are reduced to algebraic relations that constitute a system of linear equations for variances and fluxes. The solution to that system yields the expressions for the vertical fluxes of momentum and scalars in the following down-gradient form:

$$
\left\langle u_{3}^{\prime} f^{\prime}\right\rangle=-S_{f} l e^{1 / 2} \frac{\partial\langle f\rangle}{\partial x_{3}},
$$

where a generic variable $f$ stands for $u_{1}, u_{2}, \theta$ or $q$. The so-called stability functions $S_{f}$ depend on the dimensionless buoyancy gradient $\epsilon^{-2} e^{2} N^{2}$ and on the dimensionless shear $\epsilon^{-2} e^{2}\left[\left(\partial\left\langle u_{1}\right\rangle / \partial x_{3}\right)^{2}+\left(\partial\left\langle u_{2}\right\rangle / \partial x_{3}\right)^{2}\right]$. They incorporate various combinations of dimensionless coefficients that stem from the parameterisations of the pressure-velocity and pressure-scalar covariances and of the dissipation rates. The turbulence length scale $l$ is parameterised 
algebraically as discussed above. Examples of one-equation turbulence closure schemes for NWP purposes are the schemes used operationally in the limited-area model COSMO (formerly referred to as LM [187, 164, 165]) and HIRLAM [205].

At the next level of simplification, the time-rate-of-change, the advection and the turbulent diffusion of the TKE are neglected so that all secondmoment equations are reduced to algebraic relations. The resulting expressions for fluxes are essentially of the down-gradient form $\left\langle u_{3}^{\prime} f^{\prime}\right\rangle=-K_{f} \partial\langle f\rangle / \partial x_{3}$, where the diffusion coefficients $K_{f}$ are functions of the turbulence length scale and of the vertical gradients of velocity and buoyancy. These diffusion coefficients are often adjusted in a somewhat ad hoc manner in order to improve the overall performance of an NWP model (cf. the situation with mixing-length models in astrophysics discussed in chapter 3 of this volume). The algebraic turbulence closure schemes are used, for example, in the global NWP models GME [136] of the German Weather Service (DWD) and IFS (Integrated Forecasting System) [93] of the European Centre for Medium-Range Weather Forecasts (ECMWF).

Unfortunately, no simplification is possible without the sacrifice of relevant information and hence of accuracy, and this is particularly true of truncated second-order closures. Well-calibrated algebraic and one-equation turbulence closure schemes show a good performance in turbulent flows where the static stability is close to neutral. However, they are known to have serious problems in stratified flows, both stable and convective.

\section{Performance of Simplified Closures in Stratified Flows}

Turbulence in stably stratified boundary layers is weak and often intermittent in space and time [77]. The stable boundary layer (SBL) is exposed to various types of meso-scale motions, such as gravity waves and meanders of cold air, to horizontal inhomogeneity of the underlying surface, and to the radiation flux divergence. These and other effects significantly complicate the SBL structure $[135,134,62,219,183]$. Current turbulence schemes do not include many of these important effects in a physically meaningful way [133] and are not able to satisfactorily describe the SBL turbulence structure. Most current turbulence schemes tend to extinguish turbulence in case of strong static stability, when the gradient Richardson number $R i=\left[\left(\partial\left\langle u_{1}\right\rangle / \partial x_{3}\right)^{2}+\left(\partial\left\langle u_{2}\right\rangle / \partial x_{3}\right)^{2}\right]^{-1} N^{2}$ exceeds its critical value of order 0.2 (see chapter 4 of this volume for further discussion of this issue). Then, the schemes are tuned in an ad hoc way to prevent turbulence from dying out entirely as the static stability increases. A simple device often applied in NWP models is a "minimum diffusion coefficient". That is, the eddy diffusivity for momentum and scalars is limited from below by a predefined constant value to provide "residual" mixing when the turbulence parameterisation scheme predicts no turbulence at all. A tuning device of this sort may have a detrimental effect on the NWP model perfor- 
mance in some important situations. For example, it may destroy a delicate balance of physical processes (radiative and evaporative cooling, advection by mean vertical velocity, and turbulent entrainment) near the top of the stable or neutral PBL capped by stratocumulus clouds, leading to the disappearance of clouds where they should actually be maintained.

A physically plausible approach to the problem of maintaining turbulence in case of strong static stability was taken by Raschendorfer [164, 165]. He surmised that turbulence in the shear-driven SBL would not collapse entirely, if the underlying surface at the sub-grid scale is horizontally inhomogeneous with respect to the temperature. Spatial buoyancy differences due to this temperature inhomogeneity induce horizontal pressure gradients that in turn set the air in motion. Although these air motions experience friction at the underlying surface, they may not contribute to the grid-box mean momentum flux as the flow patterns in different directions may counteract each other (cf. cell-like motions in the shear-free CBL that efficiently transport heat but make no contribution to the grid-box momentum flux). However, they do contribute to the grid-scale mean TKE, preventing the SBL from collapsing entirely. Having assumed the above mechanism of maintenance of turbulence in stable stratification, Raschendorfer extended the one-equation turbulence closure scheme of the NWP model COSMO so that the scheme is guarded against sharp turbulence cut-off at a critical Richardson number.

Worthy of mention is an attempt to derive eddy viscosity in stably stratified turbulent flows from first principles made by Sukoriansky et al. [192, 190]. Their spectral model is free of the sharp cut-off critical Richardson number deficiency. It predicts turbulent eddy diffusivities for wind and scalar quantities in good agreement with observations. The new theoretical findings have been used in the framework of the two-equation $e-\epsilon$ turbulence closure scheme to model atmospheric SBL over sea ice [191].

Difficulties of simplified turbulence closure schemes in convective conditions are associated first of all with their inability to adequately account for non-local transport properties of convective turbulence. This is not particularly surprising, however, considering that in the simplified truncated closures the third-order terms largely responsible for non-local transport of momentum and scalars are either entirely neglected or parameterised very crudely. Local turbulence schemes are typically unable to reproduce the well-mixed character of the CBL with counter-gradient fluxes of scalars often encountered in the upper part of the boundary layer. They also fail to correctly represent entrainment at the CBL top, leading to erroneous prediction of the CBL temperature and humidity and of the CBL height. One way to cope with these difficulties is to introduce more of the essential physics into the secondorder closure scheme, e.g. by using the skewness-dependent formulations of third-order transport terms discussed above.

Notice that simplified truncated second-order closure schemes are almost inevitably non-realisable. In order to prevent such schemes from producing unphysical solutions, a clipping operation is usually applied. Normal stresses 
and scalar variances are set to zero if they become negative, and Schwarz' inequalities for the third-order moments are strictly enforced. The clipping operation has proven to be an effective tool [55] and is considered to be legitimate in engineering and geophysical applications [174]. It should, however, be avoided whenever possible; that is to say, effort should be mounted to develop closure schemes where clipping is reduced to a minimum.

\section{The Similarity Approach}

An alternative way to describe boundary-layer turbulence and shallow convection is through the use of the similarity theory for boundary-layer flows. The approach basically amounts to representing the vertical profiles of turbulent quantities through the shape functions, using the scales of variables pertinent to the mixing regime in question. The scaling ideas should be consistent with the budget equations for turbulence moments, at least in the integral sense (cf. the surface-layer flux-profile relationships of the Monin-Obukhov similarity theory considered in the next section).

Taking the similarity approach, shapes of the vertical profiles of the turbulent diffusion coefficients are prescribed and the magnitudes of diffusion coefficients and of other turbulence characteristics, such as the fluxes due to entrainment at the boundary layer top, are expressed through the appropriate scales of length, velocity, temperature and humidity. To this end, the now classical Deardorff convective scaling $[53,54]$ is widely used with the CBL depth $h$ as the bulk length scale, and $w_{*}=\left(h B_{s}\right)^{1 / 3}$ and $\theta_{*}=\left\langle u_{3}^{\prime} \theta^{\prime}\right\rangle_{s} / w_{*}$ as the bulk velocity and potential-temperature scales, respectively. Here, $B_{s}=\beta_{3}\left\langle u_{3}^{\prime} \theta_{v}^{\prime}\right\rangle_{s}$ is the surface buoyancy flux, and $\left\langle u_{3}^{\prime} \theta^{\prime}\right\rangle_{s}$ and $\left\langle u_{3}^{\prime} \theta_{v}^{\prime}\right\rangle_{s}$ are the surface fluxes of potential temperature and of virtual potential temperature, respectively. The humidity scale is introduced similarly to the potential-temperature scale. Powerlaw functions of dimensionless height $x_{3} / h$ are commonly utilised for the vertical-profile shape functions. For example, turbulent temperature diffusivity in shear-free CBL is expressed as $K_{\theta} / w_{*} h=C_{K \theta}\left(x_{3} / h\right)\left(1-C_{\text {entr }} x_{3} / h\right)^{\alpha}$, where $C_{K \theta}, C_{\text {entr }}$ and $\alpha$ are disposable parameters, and $C_{\text {entr }}$ is chosen so as to provide the right amount of entrainment at the CBL top. In order to account for the production of turbulence energy due to mean velocity shear, a convective velocity scale is modified through the incorporation of the surface friction velocity $u_{*}=\left(\left\langle u_{3}^{\prime} u_{1}^{\prime}\right\rangle_{s}^{2}+\left\langle u_{3}^{\prime} u_{2}^{\prime}\right\rangle_{s}^{2}\right)^{1 / 4}$, where the subscript "s" indicates the surface values. Using the similarity approach, momentum and scalar fluxes are not directly dependent on local gradients; rather they are functions of the integral scales that characterise the CBL as a whole and thus account (at least implicitly) for the non-local effects. Such a "non-local" scheme is proposed in [92], using earlier ideas presented in [201].

An advanced boundary layer mixing scheme based on the similarity approach was developed by Lock et al. ([128, 139], see also [126, 127]) for use in the UK Met Office NWP and climate models [48]. The scheme incorporates an 
entrainment parameterisation based on a generalised turbulent velocity scale that accounts for the generation of turbulence due to the mean velocity shear, due to the surface heating, due to the cloud-top radiative cooling, and due to the evaporative cooling of entrained air. The unstable layers are identified on the basis of the buoyancy of undilute parcels lifted from the surface and lowered from the cloud top with due regard for latent heat effects. The mixing regimes considered by the scheme range from dry SBL to a complex configuration, where a layer of stratocumulus clouds is separated from the unstable surface layer by a cumulus cloud layer. As different mixing parameterisations are used for different regimes, the scheme includes a sophisticated decision tree to discriminate between various boundary-layer mixing regimes. It should be noted that the scheme does not operate throughout the atmosphere. It is applied to about the lowest $2.5 \mathrm{~km}$ [128]. Mixing through the rest of the atmosphere, as well as through the cumulus cloud layers diagnosed within the area of operation of the turbulence scheme, is computed with the convection scheme [82]. Convection schemes currently used in NWP and climate models are developed on the basis of the mass-flux approach (section 3).

\subsection{The Surface Layer}

The layer in the immediate vicinity of the underlying surface, the surface layer, deserves special attention. The surface layer looms large in meteorology as it is this layer where the interaction of the atmosphere with the underlying surface takes place. In NWP and climate models, the surface-layer resistance, heat and mass transfer laws are used to compute surface fluxes of momentum, heat, water vapour, and if necessary, of other scalar quantities, and are, therefore, the key components of the physical parameterisation package.

The now classical Monin-Obukhov similarity theory $[156,152]$ has been commonly used for more than half a century to describe the vertical structure of the atmospheric surface layer. For lack of space, it is impossible to give an account of the Monin-Obukhov theory in the present paper. Readers are referred to $[63,218,153,184,67,95]$, where various aspects of the surface-layer similarity are discussed. Here we only present the Monin-Obukhov surfacelayer flux-profile relationships for the wind velocity and for the potential temperature (the formulation for specific humidity is similar to that for potential temperature). They read

$$
\begin{gathered}
u(z)-u_{s}=\frac{u_{*}}{\kappa}\left[\ln \frac{z}{z_{0 u}}+\psi_{u}(z / L)\right], \\
\theta(z)-\theta_{s}=-\operatorname{Pr}_{\mathrm{n}} \frac{\left\langle w^{\prime} \theta^{\prime}\right\rangle_{s}}{\kappa u_{*}}\left[\ln \frac{z}{z_{0 \theta}}+\psi_{\theta}(z / L)\right] .
\end{gathered}
$$

Here, $z=x_{3}$ is the height above the underlying surface, $u=u_{1}$ is the component of the wind vector along the $x_{1}$ horizontal axis that is taken to be aligned 
with the surface stress (then the wind component along the $x_{2}$ axis is zero), $w=u_{3}$ is the vertical component of the wind vector (this notation is used to stress the one-dimensionality of the approach), $u_{s}$ and $\theta_{s}$ are the values of $u$ and $\theta$, respectively, at the underlying surface ( $u_{s}$ is zero at the rigid surface), $z_{0 u}$ and $z_{0 \theta}$ are the roughness lengths with respect to wind velocity and potential temperature, respectively, and $\operatorname{Pr}_{\mathrm{n}}$ is the turbulent Prandtl number at neutral static stability. The dimensionless functions of the Monin-Obukhov similarity theory, $\psi_{u}$ and $\psi_{\theta}$, account for the effect of static stability in the surface layer. The Obukhov length [156] is defined as $L=-u_{*}^{3} /\left(\kappa B_{s}\right)$. The von Kármán constant $\kappa$ is traditionally included into the definition of $L$. At $z / L \ll 1$, i.e. in the lower part of the stratified surface layer, or throughout the surface layer in near-neutral conditions, Eqs. (27)-(28) reduce to the classical logarithmic profiles, where the roughness lengths are the principal parameters that describe the interaction of the flow with the underlying surface.

The surface-layer formulations are often presented in terms of the drag coefficient and the heat and mass transfer coefficients. One more alternative formulation is through the resistance of the surface layer to the transfer of momentum, heat and mass. The resistance is a more "physical" parameter, i.e. the parameter more directly related to the flux and the gradient of the quantity in question than the roughness length that should be viewed as a more "derived" parameter [137]. Nonetheless, the majority of the surfacelayer formulations have been given in terms of the roughness lengths and the Monin-Obukhov similarity functions, perhaps due to their convenience in representing the profiles.

There is a substantial body of literature on the Monin-Obukhov surfacelayer similarity functions. Readers are referred to the review articles [88, 89] and to the historical surveys $[38,64]$, where numerous further references can be found. As to the parameterisation of roughness lengths with respect to wind and scalar quantities (more generally, the air-land and air-sea interaction), these are discussed in $[103,35,24,67,166,106,137,68,99,225,8,33]$, to mention a few.

It should be emphasised that the Monin-Obukhov flux-profile relationships are consistent with the budget equations for the second-order turbulence moments. In essence, they represent the second-moment budgets that are truncated under the surface-layer similarity-theory assumptions. These are that (i) turbulence is continuous, stationary and horizontally-homogeneous, (ii) third-order turbulent transport is negligible, and (iii) the surface layer is a small portion of the PBL, so that the directional wind turning is negligible and turbulent fluxes can be considered approximately height-constant, equal to their surface values (in other words, changes of fluxes over the surface layer are small as compared to their changes over the entire PBL). For example, the logarithmic wind profile is readily obtained from the TKE budget equation where only the shear-production term and the dissipation term are retained. Using the surface-layer scaling relations, $e \propto u_{*}^{2}$ and $l \propto z$, to express the TKE dissipation rate through Eq. (25) along with the assumption of height- 
constant momentum flux, $\left\langle u^{\prime} w^{\prime}\right\rangle=-u_{*}^{2}$, yields the flux gradient-relationship $d\langle u\rangle / d z \propto u_{*} / z$ (the omitted proportionality constant is the reciprocal of the von Kármán constant). Its integration over $z$ results in the logarithmic wind profile. The lower limit of integration is the height $z_{0 u}$ where the wind profile extrapolated logarithmically downward approaches its surface value (zero over the rigid surface). Since the surface-layer flux-profile relationships are consistent with the (truncated) second-moment budgets, they suffer from the same shortcoming as the (truncated) second-order closures. They are known to experience problems in strongly convective and in strongly stable flows.

In conditions of free convection, when the mean wind vanishes, the surfacelayer flux-profile relationships predict zero fluxes. The failure is due to the neglect of the CBL-scale cell-like coherent motions. As the flow patterns in different directions effectively counteract each other, these motions make no contribution to the transfer of mean momentum (mean over a horizontal area that is large enough to embrace a multitude of convective cells). However, these motions efficiently transport heat and other scalar quantities. Businger [39] introduced the concept of "minimum friction velocity", that is the friction velocity due to the effect of the CBL-scale motions which do experience friction at the surface, although mean wind is zero. The minimum friction velocity was assumed to scale on the Deardorff convective velocity $w_{*}$ and to additionally depend on $z_{0 u}$. Using the above concept, a number of heat and mass transfer laws have been proposed that are suitable for calculation of surface fluxes in conditions of free convection [172, 189, 22, 194, 226, 4, 223]. Comprehensive summaries are given in $[226,223]$. Some authors used the classical $\mathrm{Nu} \propto \mathrm{Ra}^{1 / 3}$ heat transfer law to estimate surface fluxes in free convection. Notice that the Nusselt number $\mathrm{Nu}$ and the Rayleigh number Ra explicitly depend on the molecular viscosity and on the molecular heat conductivity of the medium in question. A generalisation of this law to the case of a two-component medium, e.g. moist air, was proposed in [72, 73]. Examples of its successful application to the computation of surface fluxes of sensible and latent heat are given in $[78,9]$.

Problems of the surface-layer similarity theory in conditions of strong static stability are associated with the intermittent nature of turbulence and with many other effects, such as internal gravity waves and horizontal inhomogeneity of the underlying surface, that complicate the surface-layer structure (see e.g. $[133,135,134,62,77,76])$. Traditional log-linear flux-profile relationships of the Monin-Obukhov theory predict zero fluxes as the static stability increases and the gradient Richardson number approaches its critical value. This is in conflict with most of the observational data which indicate that turbulence very often survives well above the critical Richardson number threshold and the surface fluxes of momentum and scalars are weak but non-negligible. Recall that the Monin-Obukhov flux-profile relationships are derived under a number of simplifying assumptions which restrict their limits of applicability. Their failure to describe the real-world strongly stable surface layers is not particularly surprising. Taking a pragmatic approach, the flux-profile 
relationships are adjusted in a somewhat ad hoc manner to enable the fluxes to be non-zero at sufficiently strong static stability (e.g. [24]). The study of the stably stratified PBL, including the surface layer, is a very active research area in which progress is being made and more physically justified remedial measures are proposed. Mention should be made of a series of publications by Zilitinkevich and co-authors who examined the effects of static stability at the SBL outer edge, that is characterised by the buoyancy frequency $N$, on the SBL mean and turbulence structure. The exchange of energy, both kinetic and potential, between the SBL and the overlying stably stratified atmosphere due to the radiation of internal gravity waves was analysed in $[219,183]$. Equations for the SBL depth, the SBL resistance and heat transfer laws, and the surface-layer flux-profile relationships were modified to incorporate the dependence on $N$ [229, 224, 221, 220, 222]. The surface-flux calculation algorithms were modified with due regard for the effect of $N$ and applied to determine surface fluxes of momentum and heat in numerical models of the atmosphere $[158,230,231]$.

\subsection{Extension to Saturated Air}

Up to this point the atmospheric air has been treated as unsaturated, characterised by the two thermodynamic variables, $\theta$ and $q$. The thermodynamic structure of the real atmosphere is strongly complicated by the presence of clouds. Clouds produce precipitation. They strongly interact with atmospheric radiation, changing the atmosphere energy budget, the energy budget of the underlying surface and of the PBL in particular. They also change the buoyancy of air parcels, thus affecting the rate of production/destruction of TKE by the gravitational force. All these effects related to the presence of clouds should be accurately represented in numerical models of the atmosphere. As far as the parameterisations of turbulence and of shallow non-precipitating convection are concerned, the primary goal is to account for the effect of clouds on the buoyancy production/destruction of the Reynolds stress, including its trace - the TKE, and of the scalar fluxes. The key issue is an accurate representation of the horizontal fractional cloud coverage of a given numerical-model grid box and of the amount of cloud condensate it contains [200].

In order to account for the presence of cloud condensate, turbulence and shallow-convection parameterisation schemes are formulated in terms of variables that are approximately conserved for phase changes in the absence of precipitation. Consider first warm clouds that only contain water in liquid form. Possible extension to the case of three phases including cloud ice is briefly discussed at the end of this section. One pair of moist quasi-conservative variables often used in models of non-precipitating clouds consists of the total water specific humidity $q_{t}$ and the liquid water potential temperature $\theta_{l}$ defined as $[25,56]$ 


$$
q_{t}=q+q_{l}, \quad \theta_{l}=\theta-\frac{\theta}{T} \frac{L_{v}}{c_{p}} q_{l},
$$

where $q_{l}$ is the liquid water specific humidity, $L_{v}$ is the latent heat of vapourisation, $c_{p}$ is the specific heat of air at constant pressure, and $T$ is the absolute temperature related to the potential temperature through $T=\theta\left(P / P_{0}\right)^{R_{d} / c_{p}}$, $P$ and $P_{0}$ being the atmospheric pressure and its reference value, respectively. No supersaturation is assumed, so that $q_{l}=q_{t}-q_{s}$ if $q_{t}>q_{s}$, where $q_{s}$ is the saturation specific humidity, and $q_{l}=0$ otherwise. Clearly, $q_{t}$ and $\theta_{l}$ reduce to the dry variables $q$ and $\theta$, respectively, in unsaturated conditions.

Using the above moist quasi-conservative variables, the second-moment equations (3)-(9) remain the same to within the substitution of $\theta_{l}$ and $q_{t}$ for $\theta$ and $q$, respectively. However, the buoyancy terms (the terms with $\beta_{i}$ ) in Eqs. (3), (9), (4) and (5) should be modified with due regard for the presence of cloud condensate. This problem amounts to modelling the virtual potential temperature flux $\left\langle u_{i}^{\prime} \theta_{v}^{\prime}\right\rangle$ and the scalar-virtual potential temperature covariances $\left\langle\theta_{l}^{\prime} \theta_{v}^{\prime}\right\rangle$ and $\left\langle q_{t}^{\prime} \theta_{v}^{\prime}\right\rangle$ in terms of fluctuations of $\theta_{l}$ and $q_{t}$. Using Eq. (29) and a generalised virtual potential temperature that accounts for the water loading effect $[125,15]$,

$$
\theta_{v}=\theta\left[1+(R-1) q-q_{l}\right]
$$

the above covariances are given by

$$
\begin{aligned}
\left\langle f^{\prime} \theta_{v}^{\prime}\right\rangle & =\left[1+(R-1)\left\langle q_{t}\right\rangle-R\left\langle q_{l}\right\rangle\right]\left\langle f^{\prime} \theta_{l}^{\prime}\right\rangle+(R-1)\langle\theta\rangle\left\langle f^{\prime} q_{t}^{\prime}\right\rangle \\
& +\left\{\frac{\langle\theta\rangle}{\langle T\rangle} \frac{L_{v}}{c_{p}}\left[1+(R-1)\left\langle q_{t}\right\rangle-R\left\langle q_{l}\right\rangle\right]-R\langle\theta\rangle\right\}\left\langle f^{\prime} q_{l}^{\prime}\right\rangle,
\end{aligned}
$$

where $R=R_{v} / R_{d}$, and a generic variable $f$ stands for $u_{i}, \theta_{l}$ or $q_{t}$. In order to arrive at Eq. (31), the third-order covariances and the pressure fluctuations are neglected (the latter assumption yields $\theta_{l}=\theta-\frac{\langle\theta\rangle}{\langle T\rangle} \frac{L_{v}}{c_{p}} q_{l}$ ).

In the "dry" limit, where a given numerical-model grid box is cloud free, Eq. (31) reduces to

$$
\left\langle f^{\prime} \theta_{v}^{\prime}\right\rangle_{d}=\left[1+(R-1)\left\langle q_{t}\right\rangle\right]\left\langle f^{\prime} \theta_{l}^{\prime}\right\rangle+(R-1)\langle\theta\rangle\left\langle f^{\prime} q_{t}^{\prime}\right\rangle,
$$

where $\theta_{l}$ and $q_{t}$ coincide with $\theta$ and $q$, respectively, as $q_{l}=0$. In the "wet" limit, where a given grid box is uniformly saturated, $\left\langle f^{\prime} q_{l}^{\prime}\right\rangle$ can be expressed, to a good approximation, in terms of $\left\langle f^{\prime} \theta_{l}^{\prime}\right\rangle$ and $\left\langle f^{\prime} q_{t}^{\prime}\right\rangle$ as follows:

$$
\begin{aligned}
\left\langle f^{\prime} \theta_{v}^{\prime}\right\rangle_{w} & =\left[1+(R-1)\left\langle q_{t}\right\rangle-R\left\langle q_{l}\right\rangle-\frac{\mathcal{A P}}{\mathcal{Q}}\right]\left\langle f^{\prime} \theta_{l}^{\prime}\right\rangle \\
& +\left[(R-1)\langle\theta\rangle+\frac{\mathcal{A}}{\mathcal{Q}}\right]\left\langle f^{\prime} q_{t}^{\prime}\right\rangle,
\end{aligned}
$$

where $\mathcal{A}=\frac{\langle\theta\rangle}{\langle T\rangle} \frac{L_{v}}{c_{p}}\left[1+(R-1)\left\langle q_{t}\right\rangle-R\left\langle q_{l}\right\rangle\right]-R\langle\theta\rangle, \mathcal{P}=\frac{\langle T\rangle}{\langle\theta\rangle}\left\langle q_{s l, T}\right\rangle, \mathcal{Q}=1+$ $\frac{L_{v}}{c_{p}}\left\langle q_{s l, T}\right\rangle$, and $\left.\left\langle q_{s l, T}\right\rangle \equiv \frac{\partial q_{s}}{\partial T}\right|_{T=\left\langle T_{l}\right\rangle}$ is computed from the Clausius-Clapeyron 
equation, $\frac{\partial q_{s}}{\partial T}=\frac{L_{v} q_{s}}{R_{v} T^{2}}$. A first-order Taylor expansion of the saturation specific humidity $q_{s}(T)$ about $T=\left\langle T_{l}\right\rangle$ is used to derive Eq. (33).

The simplest way to determine $\left\langle f^{\prime} \theta_{v}^{\prime}\right\rangle$ is to use either Eq. (32) or Eq. (33), assuming that a given numerical-model grid box is either all clear or all cloudy, respectively. This "all-or-nothing" approach may be used in cloud-resolving models (although some caution is still required). Since it essentially assumes no sub-grid scale fluctuations of cloud water related variables, it is not applicable in the framework of the NWP and climate models whose horizontal resolution is too coarse to resolve cloud-scale motions. As sizable SGS fluctuations of cloud water related variables exist, an expression is needed that is valid not only in the dry and wet limits, but also in the general case of fractional cloudiness. To this end, an interpolation formula is used,

$$
\left\langle f^{\prime} \theta_{v}^{\prime}\right\rangle=(1-\hat{\mathcal{R}})\left\langle f^{\prime} \theta_{v}^{\prime}\right\rangle_{d}+\hat{\mathcal{R}}\left\langle f^{\prime} \theta_{v}^{\prime}\right\rangle_{w},
$$

where $\hat{\mathcal{R}}$ is the interpolation variable satisfying $0 \leq \hat{\mathcal{R}} \leq 1$. In case the PDFs of SGS fluctuations of $\theta_{l}, q_{t}$ and $u_{3}$ (vertical velocity) are Gaussian and the fluctuations of $u_{3}$ and $q_{l}$ are uncorrelated, $\hat{\mathcal{R}}$ is identical to the fractional cloud cover $\hat{\mathcal{C}}$. In case the fluctuations of $u_{3}$ and $q_{l}$ are correlated, the PDFs can differ significantly from the Gaussians, and $\hat{\mathcal{R}}$ can deviate widely from $\hat{\mathcal{C}}$. This is the case for shallow cumuli, where the fractional cloud cover is small, $u_{3}$ and $q_{l}$ are strongly correlated, the PDFs of cloud related variables are highly skewed, and $\hat{\mathcal{R}}$ can be several times larger than $\hat{\mathcal{C}}$ [122]. In order to account for both Gaussian and non-Gaussian cases, Eq. (34) can be conveniently recast as follows:

$$
\left\langle f^{\prime} \theta_{v}^{\prime}\right\rangle=(1-\hat{\mathcal{C}})\left\langle f^{\prime} \theta_{v}^{\prime}\right\rangle_{d}+\hat{\mathcal{C}}\left\langle f^{\prime} \theta_{v}^{\prime}\right\rangle_{w}+F_{N G} \hat{\mathcal{C}}(1-\hat{\mathcal{C}})\left\langle f^{\prime} \theta_{v}^{\prime}\right\rangle_{w},
$$

where a correct behaviour in the dry $\hat{\mathcal{C}}=0$ and the wet $\hat{\mathcal{C}}=1$ limits is ensured. The deviations from the Gaussian limit are accounted for through the correction function $F_{N G}$. It is a complicated function of various cloud related quantities, such as the mean saturation deficit, variances of $\theta_{l}, q_{t}$ and $u_{3}$, and their skewness. In practice, simplified formulations of $F_{N G}$ are utilised that ignore the dependencies on some of these quantities. Usable formulations that provide a smooth transition between the Gaussian state and the nonGaussian skewed state are presented in e.g. [123, 19, 47, 20, 122].

The fractional cloud cover $\hat{\mathcal{C}}$ should now be determined. Cloud-cover parameterisation schemes proposed to date vary in terms of their complexity and physical realism. Comprehensive reviews are given in [199, 200], where further references can be found. Here, only the very basic ideas are briefly outlined.

The commonly used relative humidity schemes are termed so since they employ the grid-scale mean relative humidity $\langle R H\rangle$ as the chief predictor of the cloud cover. The sub-grid scale fluctuations of temperature and humidity enable clouds to form even though a numerical-model grid box in question is unsaturated on the average, $\langle R H\rangle<1$. A critical relative humidity $\langle R H\rangle_{\mathrm{cr}}$, 
below which $\hat{\mathcal{C}}=0$, is introduced, and the fractional cloud cover $\hat{\mathcal{C}}$ is assumed to increase monotonically with increasing $\langle R H\rangle$ until $\hat{\mathcal{C}}=1$ when $\langle R H\rangle=1$. Additional predictors, such as the grid-scale mean vertical velocity, are used in some schemes. Although the SGS variability of temperature and humidity is implicit in the relative humidity schemes, the connection between the fractional cloud cover and the SGS dynamics is rather loose.

The schemes referred to as the SGS statistical cloud schemes, pioneered by Sommeria and Deardorff [182] and Mellor [141], make use of PDFs of the SGS humidity (and temperature) fluctuations. Once the PDF is specified, the fractional cloud cover is simply the integral over a saturated part of the PDF. Since clouds can result both from the humidity fluctuations and from the temperature fluctuations, the latter ones change the local saturation vapour pressure, it is convenient to introduce, following Mellor [141], a variable $s=\mathcal{Q}^{-1}\left(\left\langle q_{t}\right\rangle-\left\langle q_{s l}\right\rangle+q_{t}^{\prime}-\mathcal{P} \theta_{l}^{\prime}\right)$, where $\left\langle q_{s l}\right\rangle=q_{s}\left(\left\langle T_{l}\right\rangle\right)$ (s here should not be confused with the dry static energy used in section 3.1$)$. The variable $s$ represents the local value of the liquid water specific humidity computed with respect to the linearised saturation specific humidity curve. This quantity has already been used above to express $\left\langle f^{\prime} q_{l}^{\prime}\right\rangle$ through $\left\langle f^{\prime} q_{t}^{\prime}\right\rangle$ and $\left\langle f^{\prime} \theta_{l}^{\prime}\right\rangle$ in Eq. (31), leading to Eq. (33). Assuming that no supersaturation occurs, the fractional cloud cover and the grid-box mean liquid water specific humidity are given by

$$
\hat{\mathcal{C}}=\int_{0}^{\infty} G(s) d s, \quad\left\langle q_{l}\right\rangle=\int_{0}^{\infty} s G(s) d s,
$$

where $G(s)$ is the PDF of $s$. If a Gaussian PDF is assumed, then $\hat{\mathcal{C}}=$ $\frac{1}{2}\left[1+\operatorname{erf}\left(\frac{\langle s\rangle}{\sqrt{2} \sigma_{s}}\right)\right]$ and $\left\langle q_{l}\right\rangle=\hat{\mathcal{C}}\langle s\rangle+\frac{\sigma_{s}}{\sqrt{2 \pi}} \exp \left(\frac{\langle s\rangle^{2}}{2 \sigma_{s}^{2}}\right)$, where erf is the error function, $\langle s\rangle=\mathcal{Q}^{-1}\left(\left\langle q_{t}\right\rangle-\left\langle q_{s l}\right\rangle\right)$ is the mean value of $s$, and $\sigma_{s} \equiv\left\langle s^{\prime 2}\right\rangle^{1 / 2}=$ $\mathcal{Q}^{-1}\left[\left\langle q_{t}^{\prime 2}\right\rangle+\mathcal{P}^{2}\left\langle\theta_{l}^{\prime 2}\right\rangle-2 \mathcal{P}\left\langle q_{t}^{\prime} \theta_{l}^{\prime}\right\rangle\right]^{1 / 2}$ is its standard deviation. Notice that $\sigma_{s}$ depends on the variances of $q_{t}$ and of $\theta_{l}$ and on their covariance. This provides an important link between the cloud cover and the dynamics of SGS motions. Apart from the Gaussian PDFs, various other PDFs have been proposed. A number of them are non-symmetric. Besides the first and the second moment of the distribution (i.e. the mean and the variance), they require higher-order moments, e.g. skewness, as an input. Tompkins [199, 200] presented a comprehensive review of the PDFs proposed by various authors and discussed several consistency issues, such as the use of statistical cloud schemes in the atmospheric models that carry a prognostic equation for $\left\langle q_{l}\right\rangle$. He also showed that there is no clear distinction between the statistical schemes and the relative humidity schemes. If the PDF moments are kept constant in space and time, the statistical cloud-cover formulations can be recast in terms of relative humidity.

Although prognostic equations may be (and often are) used to compute the PDF moments, the fractional cloud cover is determined diagnostically in 
the framework of statistical cloud schemes. Some other schemes, exemplified by the Tiedtke scheme [198], take a different approach - they determine $\hat{\mathcal{C}}$ from its own prognostic equation. Merits and shortcomings of such schemes are discussed in [81, 207, 40, 116, 200].

The $q_{t}-\theta_{l}$ system outlined above can be extended to the case of three phases including cloud ice [56]. To this end, the total water specific humidity is generalised to account for the presence of ice, $q_{t}=q+q_{l}+q_{i}$, where $q_{i}$ is the "solid water specific humidity" (the mass of cloud ice per unit mass of moist air), and the ice-liquid water potential temperature is introduced, $\theta_{i l}=$ $\theta-\frac{\theta}{T} \frac{L_{v}}{c_{p}} q_{l}-\frac{\theta}{T} \frac{L_{i}}{c_{p}} q_{i}$, where $L_{i}$ is the specific heat of sublimation. The saturation specific humidity requires a generalised definition, the simplest of which is $q_{s}=\left(1-\mathcal{F}_{i}\right) q_{s l}+\mathcal{F}_{i} q_{s i}$, where $\mathcal{F}_{i}=q_{i} /\left(q_{l}+q_{i}\right)$ is the cloud-ice fraction of the total cloud condensate, and $q_{s l}$ and $q_{s i}$ are the saturation specific humidity for the vapour-liquid equilibrium and for the vapour-ice equilibrium, respectively. If ice and liquid water are allowed to co-exist over a certain temperature range with a finite rate of liquid-ice transformation of one into the other, a simple function of temperature can be used to determine $\mathcal{F}_{i}$. Alternatively, a rate equation for $\mathcal{F}_{i}$ can be employed [56]. The use of the $q_{t}-\theta_{i l}$ system raises various issues, such as allowance for supersaturation and consistency with the prognostic equations for $\left\langle q_{i}\right\rangle$ and its precipitating components that are carried by many atmospheric models. Theses issues necessitate an extensive discussion that is beyond the scope of the present paper.

In closing this section it should be emphasised that an accurate prediction of the fractional cloud cover and of the amount of cloud condensate is of great importance for radiation calculations. The SGS cloud scheme is thus an essential component of the physical parameterisation package of an atmospheric model that provides a tight coupling between various parameterisation schemes.

\section{Mass-Flux Convection Schemes}

This section discusses the mass-flux modelling framework that is widely used to parameterise convection, both deep precipitating and shallow nonprecipitating, in numerical models of the atmosphere. First, the most salient features of the mass-flux convection schemes, as they are currently used in NWP and related applications, are recollected. Then, the analogy between budget equations for the second-order moments of fluctuating fields derived within the mass-flux modelling framework and within the ensemble-mean second-order modelling framework are examined. These exercises help to elucidate the physical meaning of some closure assumptions and disposable parameters of mass-flux schemes. They also demonstrate the similarities and the differences between the two approaches and suggest how the mass-flux parameterisations can be formulated in terms of the ensemble-mean second-order closures and vice versa. The analysis of the second-order moment budgets 
is performed using the simplest "two-delta-function" mass-flux framework. Most currently used mass-flux schemes are (formally) based on a slightly more complex "three-delta-function" framework. However, the use of a simplified two-delta-function framework does not affect the principal results from the analysis.

\subsection{Outline of Basic Features}

In this section, the basic features of the mass-flux convection schemes are outlined. Attention is focused on the scheme developed by Tiedtke [197] (hereafter T89). That scheme is taken as an example as it was the first comprehensive mass-flux scheme that found a wide utility in NWP and climate modelling. Other mass-flux convection schemes have been developed to date, as for instance, the Kain-Fritsch scheme [97, 98, 96], the Gregory and Rowntree scheme [82], and the scheme used in the IFS of ECMWF [93, 18]. Further examples are the schemes proposed by Emanuel [59] and by Bechtold et al. [17]. Although various mass-flux schemes differ from the T89 scheme in many details, they rest on the same basic assumptions. Early ideas regarding the parameterisation of convection in atmospheric models, including the moisture convergence schemes (e.g. [107, 108]), convective adjustment schemes (e.g. [26, 27]), and mass-flux schemes (e.g. [13, 32]), are discussed in [65, 196, 12]. A comparative analysis of several cumulus parameterisation schemes is given in [186].

The T89 scheme, as well as its derivatives, utilises a triple top-hat decomposition. A fluctuating quantity in question is represented as

$$
\bar{f}=a_{u} f_{u}+a_{d} f_{d}+a_{e} f_{e},
$$

where a generic variable $f$ refers to the vertical velocity $w$, the dry static energy per unit mass $s=c_{p} T+g z$, the specific humidity $q$, the specific cloud water content $q_{l}$, or to any other quantity treated by a parameterisation scheme. We focus attention on the mass-flux parameterisation of scalar transport. Momentum transport by convection and its parameterisation through the massflux approach are considered in e.g. [102, 80]. The notation with $w=u_{3}$, $z=x_{3}$ and $g=g_{3}$ is used in this section to stress the one-dimensionality of the approach, where only vertical convective transport is considered. An overbar denotes a horizontal mean, and the subscripts "u", "d" and "e" refer to the contribution from convective updraughts, from convective downdraughts, and from the environmental air, respectively. The fractional areas of updraughts, $a_{u}$, of downdraughts, $a_{d}$, and of environmental air, $a_{e}$, satisfy $a_{u}+a_{d}+a_{e}=1$.

Notice that a coherent top-hat part of the quantity in question does not contain residual "sub-plume" fluctuations. Hence, the moments of fluctuating fields in the mass-flux approximation do not generally coincide with the moments in the ensemble framework (although they may by close to each other if the coherent part dominates). An overbar is used to denote quantities in the mass-flux approximation. 
Although the T89 scheme is formally based on the triple decomposition (37), the properties of environmental air do not appear in the governing equations. The reason is that a mean over the environment is taken to be equal to a horizontal mean. For scalar quantities, this means $s_{e}=\bar{s}, q_{e}=\bar{q}$ and $q_{l e}=\overline{q_{l}}$. Assuming further that updraughts and downdraughts are in a steady state, the T89 scheme solves a number of ordinary differential equations (in the vertical co-ordinate) for the mass fluxes and for the fluxes of scalar quantities in convective updraughts and convective downdraughts. The equations for convective updraughts read

$$
\begin{gathered}
\frac{\partial M_{u}}{\partial z}=E_{u}-D_{u} \\
\frac{\partial}{\partial z} M_{u} X_{u}=E_{u} \bar{X}-D_{u} X_{u}+\bar{\rho} a_{u} F_{x u}
\end{gathered}
$$

where $M_{u}$ is the updraught mass flux defined as

$$
M_{u}=\bar{\rho} a_{u}\left(w_{u}-\bar{w}\right),
$$

$\rho$ is the density, and $E_{u}$ and $D_{u}$ are the rates of mass entrainment and detrainment per unit length. In order to closely follow the nomenclature traditionally used in the description of mass-flux schemes, the density appears explicitly in the equations of this section. A scalar $X$ stands for $s, q$ or $q_{l}$, and $F_{x}$ stands for the source of the scalar $X$ due to condensation/evaporation and precipitation fall-out. Similar equations are formulated for convective downdraughts, except that liquid water flux is zero. The problem is closed through the use of several parameterisation rules to specify the vertical extent of convection, the fluxes through the cloud base and the cloud top, the type of convection (penetrative, mid-level or shallow), and the rates of entrainment and detrainment.

The vertical extent of convection is specified using the parcel method (see e.g. [175]). The fluxes through the cloud base are related to the moisture convergence in the sub-cloud layer (convergence of moisture fluxes due to both resolved-scale and sub-grid scale motions), as is the case in the original T89 scheme. An alternative formulation is through the convective available potential energy (CAPE), as, for example, in the ECMWF IFS convection scheme [93] in case of deep convection. The entrainment and detrainment rates are split into two parts, turbulent entrainment/detrainment through the cloud edges (lateral boundaries) and organised entrainment/detrainment through the cloud edges and through the cloud base and the cloud top. The organised entrainment through the updraught edges is set proportional to the large-scale moisture convergence. It is only considered for penetrative and mid-level convection and for the layer from the cloud base up to the level of strongest vertical ascent. The organised detrainment of updraughts occurs at the cloud top. The organised detrainment of downdraughts occurs in the sub-cloud layer. The rates of turbulent entrainment and detrainment are set 
proportional to the mass flux, that is $E_{u}=\epsilon_{u} M_{u}$ and $D_{u}=\delta_{u} M_{u}$ for the updraughts (similar for the downdraughts), where $\epsilon_{u}$ and $\delta_{u}$ are simply taken to be constant (different for different types of convection).

The simplifying assumptions of the T89 scheme, as well as of other massflux convection schemes, are many and varied. The two crucial assumptions have already been mentioned above: (i) convection is in a quasi-steady state and (ii) the mean over the environment is equal to the mean over a grid box. Several other points should be emphasised at once. First, although convective motions are driven by buoyancy, the buoyancy term is not explicitly present in Eq. (38). Second, neither Eq. (38) for the mass flux nor Eq. (39) for the flux of a scalar contains pressure terms. In ensemble-mean second-order closure models, the pressure-velocity and pressure-scalar correlations appear explicitly (see section 2). The fact that these terms do not appear explicitly in mass-flux equations suggests that the other terms serve to perform their function. This issue is discussed below. The third point to note is that the updraught fraction $a_{u}$ and the updraught vertical velocity $w_{u}$ are not estimated separately - only their combination, namely, the updraught mass flux $M_{u}$, is computed through Eqs. (38) and (40).

Some mass-flux convection schemes make use of an equation for the updraught kinetic energy. It reads (see e.g. [93])

$$
\frac{1}{2} \frac{\partial w_{u}^{2}}{\partial z}=\frac{1}{C_{k 1}\left(1+C_{k 2}\right)} g \frac{T_{v u}-\bar{T}}{\bar{T}}-\frac{\mu_{u}}{M_{u}}\left(1+C_{k 3} C_{k 4}\right) w_{u}^{2},
$$

where $T_{v}$ is the virtual temperature, and $C_{k 1}, C_{k 2}, C_{k 3}$ and $C_{k 4}$ are empirical dimensionless constants. The "mixing coefficient" $\mu_{u}$ is set equal to either $E_{u}$ or $D_{u}$, whichever is larger. The vertical acceleration on the left-hand side of Eq. (41) is a difference between a buoyancy force and a drag force represented by the first term and the second term on the r.h.s. of Eq. (41), respectively. Equation (41) originates from the work of Simpson et al. ([178], see also [179, 79]). It is meant to describe the vertical acceleration of a cumulus tower that is treated as an idealised jet, a buoyant rising thermal, or a "thermal" with

vortical internal circulation. The tower kinetic energy is given by $\frac{1}{2} w_{u}^{2}$. In this respect, it is not clear if Eq. (41) is a good approximation to the budget equation for the kinetic energy of convective motions averaged over a grid box of a numerical model.

\subsection{The Two-Delta-Function Mass-Flux Framework}

In this section, the conventional two-delta-function mass-flux framework that has been widely used to parameterise atmospheric convection, shallow cumulus convection in particular, is briefly described. A more detailed account of the mass-flux framework is given in [163, 52, 111, 112, 83].

In the two-delta-function mass-flux framework, a fluctuating quantity in question is represented as 


$$
\bar{f}=a_{u} f_{u}+a_{d} f_{d}
$$

where the subscripts " $u$ " and "d" refer to contributions from convective updraughts and convective downdraughts, respectively. The fractional areas of updraughts, $a_{u}$, and downdraughts, $a_{d}$, satisfy $a_{u}+a_{d}=1$. The decomposition (42) can be formulated in terms of the probabilities of convective motions to be either updraughts, $P_{u}$, or downdraughts, $P_{d}$, so that $P_{u}+P_{d}=1$ (e.g. $[210,227,147])$. The downdraught in the two-delta-function mass-flux framework should not be confused with the downdraught in the three-delta-function framework. Equation (37) reduces to Eq. (42) if the downdraught and the environment in the triple decomposition are treated together.

All moments of fluctuating fields in the mass-flux approximation are computed through the following averaging rule:

$$
\begin{aligned}
& \overline{w^{\prime n} X^{\prime m}}=a\left(w_{u}-\bar{w}\right)^{n}\left(X_{u}-\bar{X}\right)^{m}+(1-a)\left(w_{d}-\bar{w}\right)^{n}\left(X_{d}-\bar{X}\right)^{m} \\
& =a(1-a)\left[(1-a)^{m+n-1}-(-a)^{m+n-1}\right]\left(w_{u}-w_{d}\right)^{n}\left(X_{u}-X_{d}\right)^{m},
\end{aligned}
$$

where $a=a_{u}$ and $1-a=a_{d}$. According to Eq. (43), the flux of a quantity $X$ is given by

$$
\begin{gathered}
\overline{w^{\prime} X^{\prime}}=a\left(w_{u}-\bar{w}\right)\left(X_{u}-\bar{X}\right)+(1-a)\left(w_{d}-\bar{w}\right)\left(X_{d}-\bar{X}\right) \\
=a(1-a)\left(w_{u}-w_{d}\right)\left(X_{u}-X_{d}\right)=\frac{M_{c}}{\bar{\rho}}\left(X_{u}-X_{d}\right),
\end{gathered}
$$

where $M_{c}$ is the convective mass flux introduced in [163],

$$
M_{c}=\bar{\rho} a(1-a)\left(w_{u}-w_{d}\right) .
$$

The vertical-velocity variance and the scalar variance are given by

$$
\overline{w^{\prime 2}}=a(1-a)\left(w_{u}-w_{d}\right)^{2}, \quad \overline{X^{\prime 2}}=a(1-a)\left(X_{u}-X_{d}\right)^{2},
$$

and the third-order moments are given by

$$
\begin{gathered}
\overline{w^{\prime 3}}=a(1-a)(1-2 a)\left(w_{u}-w_{d}\right)^{3}, \\
\overline{X^{\prime 3}}=a(1-a)(1-2 a)\left(X_{u}-X_{d}\right)^{3}, \\
\overline{w^{\prime 2} X^{\prime}}=a(1-a)(1-2 a)\left(w_{u}-w_{d}\right)^{2}\left(X_{u}-X_{d}\right), \\
\overline{w^{\prime} X^{\prime 2}}=a(1-a)(1-2 a)\left(w_{u}-w_{d}\right)\left(X_{u}-X_{d}\right)^{2} .
\end{gathered}
$$


The following distinctive features of Eqs. (44)-(50) should be emphasised. All second-order and third-order moments vanish in the limiting cases of $a=0$ and of $a=1$. The former case is merely the case of no convection. The case $a=1$ corresponds to a convective updraught that covers the entire horizontal area in question, e.g. the entire grid box of a numerical model. Then, the updraught is no longer a sub-grid scale feature. With $a=1$ it becomes a grid scale feature that should be described by the evolution equations for the resolved fields. Notice that this is not the case for the T89 and similar massflux convection schemes. Due to their implicit assumption that $a \ll 1$, those convection schemes remain active no matter how large/small the horizontal size of a grid box of a numerical model as compared to the size of an updraught. The lack of sensitivity to the grid size becomes a serious problem as the resolution of numerical models is increased.

The r.h.s. of Eqs. (47)-(50) contain a factor $1-2 a$, i.e. the third-order moments vanish as $a=1 / 2$. The value of $a=1 / 2$ corresponds to zero skewness. It is readily shown using Eqs. (46) and (47) that the vertical-velocity skewness is given by $S_{w}=[a(1-a)]^{-1 / 2}(1-2 a)$. Notice that in the framework of the simplest mass-flux approach considered here the magnitude of the skewness $S_{x}$ of a scalar field $X$ is the same as the magnitude of $S_{w}$. An extended twoscale mass-flux framework [83] enables $S_{x}$ to be different in magnitude from $S_{w}$. The fact that the third-order moments vanish if the sub-grid scale velocity and scalar fields are not skewed is accounted for by the expressions (19) and (20) discussed in section 2.2. In this regard, Eqs. (19) and (20) are nothing but the mass-flux Eqs. (49) and (50) recast in terms of the ensemble-mean quantities used in the second-order closure approach.

The budget equations for the updraught and for the downdraught are (see e.g. $[52,111])$

$$
\begin{gathered}
\frac{\partial}{\partial t} \bar{\rho} a X_{u}+\frac{\partial}{\partial z} \bar{\rho} a w_{u} X_{u}=E X_{d}-D X_{u}+\bar{\rho} a F_{x u} \\
\frac{\partial}{\partial t} \bar{\rho}(1-a) X_{d}+\frac{\partial}{\partial z} \bar{\rho}(1-a) w_{d} X_{d}=D X_{u}-E X_{d}+\bar{\rho}(1-a) F_{x d}
\end{gathered}
$$

where $E(D)$ is the lateral mass exchange rate from the sinking (rising) fluid into the rising (sinking) fluid.

Setting $X_{u}=X_{d}=1$ and $F_{x u}=F_{x d}=0$ in Eqs. (51) and (52) yields

$$
\begin{gathered}
\frac{\partial}{\partial t} \bar{\rho} a+\frac{\partial}{\partial z} \bar{\rho} a w_{u}=E-D, \\
\frac{\partial}{\partial t} \bar{\rho}(1-a)+\frac{\partial}{\partial z} \bar{\rho}(1-a) w_{d}=D-E .
\end{gathered}
$$

Adding Eqs. (53) and (54) gives the continuity equation for the mean flow, 


$$
\frac{\partial \bar{\rho}}{\partial t}+\frac{\partial}{\partial z} \bar{\rho} \bar{w}=0
$$

Multiplying Eq. (54) by $a$ and subtracting the result from Eq. (53) times $1-a$ yields the equation that relates the fractional area of the updraught with the mass-flux divergence and with the entrainment and detrainment rates. It reads

$$
\begin{aligned}
\bar{\rho}\left(\frac{\partial}{\partial t}+\bar{w} \frac{\partial}{\partial z}\right) a & =-\frac{\partial}{\partial z} \bar{\rho} a(1-a)\left(w_{u}-w_{d}\right)+E-D \\
& =-\frac{\partial M_{c}}{\partial z}+E-D .
\end{aligned}
$$

Notice an essential difference to Eq. (38) where the substantial derivative of $a$ is neglected. This neglect deprives the mass-flux convection schemes of memory, making the vertical profile of mass flux to adjust instantaneously to the current state of the atmosphere.

Adding Eqs. (51) and (52) and rearranging gives the equation for $\bar{X}$ in the mass-flux approximation,

$$
\bar{\rho}\left(\frac{\partial}{\partial t}+\bar{w} \frac{\partial}{\partial z}\right) \bar{X}=-\frac{\partial}{\partial z} M_{c}\left(X_{u}-X_{d}\right)+\bar{\rho} \overline{F_{x}} .
$$

A direct analogy to the ensemble-mean equation for mean scalar concentration is immediately recognised. The first term on the r.h.s. of Eq. (57) is the massflux analogue of the turbulent scalar flux divergence term in the ensemblemean equation.

\subsection{Analogies between the Mass-Flux and the Ensemble-Mean Second-Moment Budgets}

In this section, analogies between the mass-flux and the ensemble-mean budget equations for the second-order moments are examined. The budgets of the scalar variance, of the vertical-velocity variance and of the vertical scalar flux are considered. It should be mentioned that these budget equations are not explicitly carried by most of the mass-flux models developed to date. Their consideration is, however, required in order to elucidate the physical meaning of the various terms in the mass-flux model equations. An analysis of the scalar-variance equations has been previously performed by de Roode et al. [52] and Lappen and Randall [111]. They found, among other things, that the sum of the lateral entrainment and detrainment rates in the mass-flux equation corresponds to the inverse scalar-variance dissipation time scale in the ensemble-mean equation. For the sake of clarity and completeness, the treatment of the scalar-variance budget is repeated here. We then extend the analysis of de Rode et al. and of Lappen and Randall to examine the budgets of the vertical-velocity variance and of the vertical scalar flux, giving particular attention to the role of the pressure-velocity and pressure-scalar covariances. The two-delta-function framework is used for the analysis. The use of the three-delta-function framework would make derivations more cumbersome, but would not affect the results in a principal way. 


\section{Scalar Variance}

Subtracting Eq. (52) times $a$ from Eq. (51) times $1-a$, then multiplying the result by $X_{u}-X_{d}$ and rearranging yields the budget equation for the scalar variance in the mass-flux approximation. Omitting algebraic manipulations, we obtain

$$
\begin{gathered}
\frac{1}{2}\left(\frac{\partial}{\partial t}+\bar{w} \frac{\partial}{\partial z}\right) a(1-a)\left(X_{u}-X_{d}\right)^{2}= \\
-\frac{M_{c}}{\bar{\rho}}\left(X_{u}-X_{d}\right) \frac{\partial \bar{X}}{\partial z}-\frac{1}{2 \bar{\rho}} \frac{\partial}{\partial z}(1-2 a) M_{c}\left(X_{u}-X_{d}\right)^{2} \\
-\frac{E+D}{2 \bar{\rho}}\left(X_{u}-X_{d}\right)^{2}+a(1-a)\left(X_{u}-X_{d}\right)\left(F_{x u}-F_{x d}\right) .
\end{gathered}
$$

This equation should be compared with the ensemble-mean budget equation for the scalar variance,

$$
\begin{gathered}
\frac{1}{2}\left(\frac{\partial}{\partial t}+\langle w\rangle \frac{\partial}{\partial z}\right)\left\langle X^{\prime 2}\right\rangle= \\
-\left\langle w^{\prime} X^{\prime}\right\rangle \frac{\partial\langle X\rangle}{\partial z}-\frac{1}{2} \frac{\partial}{\partial z}\left\langle w^{\prime} X^{\prime 2}\right\rangle-\epsilon_{x}+\left\langle X^{\prime} F_{x}^{\prime}\right\rangle .
\end{gathered}
$$

The terms on the r.h.s. of Eq. (59) represent the mean-gradient production/destruction, turbulent transport, the dissipation rate of the scalar variance, and the source term. Except for the source term, Eq. (59) is simply a one-dimensional form of Eqs. (6) and (7).

A comparison shows that there is a direct analogy between the first, the second and the fourth terms on the r.h.s. of the mass-flux and of the ensemblemean equations. The third term on the r.h.s. of the mass-flux equation (58) is negative definite. It acts to decrease the scalar difference between the updraught and the downdraught and can, by analogy with the third term on the r.h.s. of the ensemble-mean equation (59), be interpreted as the scalar-variance dissipation. Then, the quantity $2 a(1-a) \bar{\rho} /(E+D)$ in the mass-flux equation corresponds to the scalar-variance dissipation time scale $\tau_{\epsilon x} \equiv\left\langle X^{\prime 2}\right\rangle / \epsilon_{x}$ in the ensemble-mean equation. It is worth noting $[52,111]$ that the mass-flux "dissipation" term does not originate directly from the molecular diffusion term in the scalar equation. This term originates from the lateral exchange terms in the updraught-downdraught model and from their parameterisation through the entrainment-detrainment concept. The above analogy is useful as it guides the way to set the rates of entrainment and detrainment in the mass-flux models. These quantities should be parameterised so as to provide the most realistic scalar-variance dissipation rate. Further requirements are imposed by the budgets of the vertical-velocity variance and of the vertical scalar flux. 


\section{Vertical-Velocity Variance}

The budget equations for the vertical velocity in the updraught and in the downdraught are

$$
\begin{gathered}
\frac{\partial}{\partial t} \bar{\rho} a w_{u}+\frac{\partial}{\partial z} \bar{\rho} a w_{u} w_{u}= \\
-a(\partial p / \partial z)_{u}-\bar{\rho} a g \frac{\theta_{r}-\theta_{u}}{\theta_{r}}+E w_{d}-D w_{u} \\
\frac{\partial}{\partial t} \bar{\rho}(1-a) w_{d}+\frac{\partial}{\partial z} \bar{\rho}(1-a) w_{d} w_{d}= \\
-(1-a)(\partial p / \partial z)_{d}-\bar{\rho}(1-a) g \frac{\theta_{r}-\theta_{d}}{\theta_{r}}+D w_{u}-E w_{d}
\end{gathered}
$$

where $p$ is the deviation of pressure (here not divided by density) from its reference value in hydrostatic equilibrium. To simplify notation, $\theta$ is used in the buoyancy terms instead of $\theta_{v}$.

Adding Eqs. (60) and (61), using Eqs. (42), (55) and (56) and rearranging gives the equation for $\bar{w}$,

$$
\bar{\rho}\left(\frac{\partial}{\partial t}+\bar{w} \frac{\partial}{\partial z}\right) \bar{w}=-\overline{\partial p / \partial z}-\bar{\rho} g \frac{\theta_{r}-\bar{\theta}}{\theta_{r}}-\frac{\partial}{\partial z} M_{c}\left(w_{u}-w_{d}\right),
$$

where the third term on the r.h.s. is the mass-flux analogue of the Reynolds stress divergence term in the ensemble-mean equation.

Equations (60), (61), and (62) for the vertical velocity are similar to Eqs. (51), (52), and (57) for a scalar except that the equations for $w$ contain the pressure-gradient and the buoyancy terms, the first and the second terms on the r.h.s. of Eqs. (60), (61), and (62), respectively. ${ }^{4}$ The updraughtdowndraught decomposition of the buoyancy term presents no difficulties. The treatment of the pressure terms in the mass-flux framework is tricky and requires special consideration.

First and foremost we emphasise that the updraught-downdraught decomposition cannot be applied to the pressure itself. A straightforward decomposition of $\bar{p}$ through Eq. (42) assumes a zero-order pressure jump across the updraught-downdraught interface that would result in a spurious source term in the equation for the vertical-velocity variance. A rigorous way to go is to take the divergence of the momentum equation and to solve the resulting

${ }^{4}$ In our formulation, there is no source term in the vertical momentum equation. A more rigorous formulation, including momentum changes due to the presence of hydrometeors (e.g. rain and snow), is given in [14]. 
Poisson equation for the fluctuating pressure in terms of the Green's function (see section 2.2). The pressure field so obtained would be consistent with the governing momentum and scalar equations.

We take a different approach. It is based on the observation that the pressure terms are actually not explicitly considered at all in the mass-flux models. ${ }^{5}$ For example, the updraught mass flux and the updraught fluxes of scalar quantities in the T89 scheme are computed on the basis of Eqs. (38) and (39), where the scalar source terms account for the effects of condensation/evaporation and of precipitation fall-out. In this way no pressure effects are explicitly accounted for. This is apparently because the mass continuity within the mass-flux framework is assumed to be satisfied exactly from the very outset. Even so, the pressure effects should be implicitly accounted for in the mass-flux second-moment budgets, and it remains to be seen which terms in the budgets serve this function. From the above line of reasoning, the pressure terms in Eqs. (60), (61) and (62) should be set to zero so that they do not appear in their explicit form in the mass-flux second-moment budgets.

The equation for the vertical-velocity variance is derived in the same way as the equation for the scalar variance. Subtracting Eq. (61) times $a$ from Eq. (60) times $1-a$, then multiplying the result by $w_{u}-w_{d}$ and rearranging, we obtain the budget equation for the vertical-velocity variance in the massflux approximation. It reads

$$
\begin{gathered}
\frac{1}{2}\left(\frac{\partial}{\partial t}+\bar{w} \frac{\partial}{\partial z}\right) a(1-a)\left(w_{u}-w_{d}\right)^{2}=-\frac{M_{c}}{\bar{\rho}}\left(w_{u}-w_{d}\right) \frac{\partial \bar{w}}{\partial z}+\frac{g}{\theta_{r}} \frac{M_{c}}{\bar{\rho}}\left(\theta_{u}-\theta_{d}\right) \\
-\frac{1}{2 \bar{\rho}} \frac{\partial}{\partial z}(1-2 a) M_{c}\left(w_{u}-w_{d}\right)^{2}-\frac{E+D}{2 \bar{\rho}}\left(w_{u}-w_{d}\right)^{2}
\end{gathered}
$$

We have omitted algebraic manipulations leading to Eq. (63) as they are fairly straightforward. Equation (63) should be compared with the ensemble-mean budget equation for the vertical-velocity variance [cf. Eq. (3)],

$$
\begin{gathered}
\frac{1}{2}\left(\frac{\partial}{\partial t}+\langle w\rangle \frac{\partial}{\partial z}\right)\left\langle w^{\prime 2}\right\rangle=-\left\langle w^{\prime 2}\right\rangle \frac{\partial\langle w\rangle}{\partial z}+\frac{g}{\theta_{r}}\left\langle w^{\prime} \theta^{\prime}\right\rangle \\
-\frac{1}{2} \frac{\partial}{\partial z}\left\langle w^{\prime 3}\right\rangle-\frac{1}{\bar{\rho}}\left\langle w^{\prime} \partial p^{\prime} / \partial z\right\rangle-\epsilon_{w},
\end{gathered}
$$

${ }^{5}$ Strictly speaking, the pressure terms are not present in the overwhelming majority of the mass-flux models developed to date. An exception is the mass-flux model ADHOC developed by Lappen and Randall [111, 112, 113]. However, the parameterisation of the pressure terms in ADHOC is based on the ensemble-mean second-order closure ideas. A new version of ADHOC, ADHOC2 [114], incorporates a representation of the pressure terms that is consistent with the mass-flux framework [115]. Earlier attempts to account for the effect of perturbation pressure on cumulus convection are reported in e.g. [90, 213, 208]. 
where the terms on the r.h.s. represent the mean-gradient production/destruction, the buoyancy production/destruction, turbulent transport, the vertical velocity-pressure gradient covariance, and the dissipation rate of the verticalvelocity variance.

Comparing Eqs. (63) and (64), we conclude that there is a direct analogy between the first, the second and the third terms on the r.h.s. of the mass-flux and the ensemble-mean equations. As to the last term on the r.h.s. of Eq. (63), two interpretations can be suggested.

We recall (see section 2.1) that it is common practice within the ensemblemean second-order modelling framework to separate out the pressure transport from the pressure gradient-velocity correlation [represented by the fourth term on the r.h.s. of Eq. (64)] and to model pressure transport together with the turbulent transport [the divergence of the third-order velocity correlation represented by the third term on the r.h.s. of Eq. (64)]. The rest of the pressure term (pressure redistribution) is modelled separately. It is usually decomposed into the rapid part and the slow part, where the slow part is believed to return turbulence toward isotropy (section 2.2). Numerous studies have revealed the importance of pressure terms in maintaining the second-moment budgets in turbulent flows. Inadequate modelling of pressure terms, the pressure redistribution in particular, most often results in inaccurate prediction of fluxes and variances and consequently of the mean fields.

Assume that no account whatsoever is taken of the pressure effects in the mass-flux framework. Then the last term on the r.h.s. of the mass-flux equation (63) can be interpreted as the dissipation of the vertical-velocity variance. This term acts to decrease the vertical-velocity difference between the updraught and the downdraught and is negative definite. With no pressure terms in the mass-flux budget, the transport of variance is solely due to the thirdorder velocity correlation, and the pressure redistribution is not accounted for. In convective flows, both pressure transport and pressure redistribution are known to be substantial. Their neglect results in a deficient vertical-velocity variance budget.

Another possible interpretation of the last term on the r.h.s. of Eq. (63) can be offered by assuming that, although the pressure term is not explicitly considered in the mass-flux budget, the pressure effects are implicitly accounted for. Then, the last term on the r.h.s. of (63) should describe the combined effect of the dissipation and of the pressure redistribution. In most convective flows, the pressure redistribution acts to reduce the vertical-velocity variance. This is explained from the following simple reasoning. The major source of energy in convection is the buoyancy that directly feeds the vertical component of the fluctuating velocity. The horizontal components of the fluctuating velocity are not fed directly. They grow at the expense of the vertical velocity component. By this means turbulence is driven toward isotropy. It is the traceless part of the pressure gradient-velocity correlation, i.e. the pressure redistribution term, that accounts for the inter-component energy exchange. Since both the dissipation and the pressure redistribution tend to reduce the vertical-velocity 
variance, it is reasonable to assume that the last term on the r.h.s. of Eq. (63) accounts for their combined effect. A problem, however, arises if we attempt to reconcile the last term in the vertical-velocity variance budget (63) with a similar term in the scalar variance budget (58). The term with $E+D$ in the scalar variance budget describes the scalar variance dissipation, whereas a similar term in the vertical-velocity variance budget describes the combined effect of the dissipation and of the pressure redistribution. Putting it differently, the same quantity $2 a(1-a) \bar{\rho} /(E+D)$ should characterise both the scalar-variance relaxation time scale due to dissipation and the velocity-variance relaxation time scale due to both dissipation and pressure redistribution. Further difficulties are encountered with the mass-flux budget equation for the vertical scalar flux.

\section{Scalar Flux}

Subtracting Eq. (52) times $a$ from Eq. (51) times $1-a$ and rearranging gives the equation for the updraught-downdraught scalar difference $X_{u}-X_{d}$. Similar manipulations with Eq. (61) and (60) (recall that there are no explicit pressure terms in the mass-flux equations) gives the equation for the updraughtdowndraught vertical-velocity difference $w_{u}-w_{d}$. Then, adding the equation for $X_{u}-X_{d}$ multiplied by $w_{u}-w_{d}$ and the equation for $w_{u}-w_{d}$ multiplied by $X_{u}-X_{d}$ and rearranging, we obtain the budget equation for the vertical scalar flux in the mass-flux approximation. Omitting algebraic manipulations, we obtain

$$
\begin{gathered}
\left(\frac{\partial}{\partial t}+\bar{w} \frac{\partial}{\partial z}\right) a(1-a)\left(w_{u}-w_{d}\right)\left(X_{u}-X_{d}\right)= \\
-\frac{M_{c}}{\bar{\rho}}\left(w_{u}-w_{d}\right) \frac{\partial \bar{X}}{\partial z}-\frac{M_{c}}{\bar{\rho}}\left(X_{u}-X_{d}\right) \frac{\partial \bar{w}}{\partial z} \\
+a(1-a) \frac{g}{\theta_{r}}\left(\theta_{u}-\theta_{d}\right)\left(X_{u}-X_{d}\right)-\frac{1}{\bar{\rho}} \frac{\partial}{\partial z}(1-2 a) M_{c}\left(w_{u}-w_{d}\right)\left(X_{u}-X_{d}\right) \\
-\frac{E+D}{\bar{\rho}}\left(w_{u}-w_{d}\right)\left(X_{u}-X_{d}\right)+a(1-a)\left(w_{u}-w_{d}\right)\left(F_{x u}-F_{x d}\right) .
\end{gathered}
$$

Equation (65) should be compared with the ensemble-mean budget equation for the vertical scalar flux [cf. Eqs. (4) and (5)],

$$
\left(\frac{\partial}{\partial t}+\langle w\rangle \frac{\partial}{\partial z}\right)\left\langle w^{\prime} X^{\prime}\right\rangle=-\left\langle w^{\prime 2}\right\rangle \frac{\partial\langle X\rangle}{\partial z}-\left\langle w^{\prime} X^{\prime}\right\rangle \frac{\partial\langle w\rangle}{\partial z}
$$




$$
+\frac{g}{\theta_{r}}\left\langle\theta^{\prime} X^{\prime}\right\rangle-\frac{\partial}{\partial z}\left\langle w^{\prime 2} X^{\prime}\right\rangle-\frac{1}{\bar{\rho}}\left\langle X^{\prime} \partial p^{\prime} / \partial z\right\rangle+\left\langle w^{\prime} F_{x}^{\prime}\right\rangle,
$$

where the terms on the r.h.s. represent the mean-gradient production/destruction (the first two terms on the r.h.s.), the buoyancy production/destruction, turbulent transport, the pressure gradient-scalar covariance, and the source term.

There is a direct analogy between all terms in the mass-flux and the ensemble-mean budgets, except the previous last terms. The previous last term on the r.h.s. of the mass-flux equation (65) is purely destructive. It acts to decrease the magnitude of the vertical scalar flux. Its counterpart in the ensemble-mean equation (66) is, however, not the dissipation term, but the pressure gradient-scalar covariance. In high Reynolds number flows (with local isotropy at small scales, see section 2.2), molecular dissipation of scalar fluxes is negligible, and it is the pressure gradient-scalar covariance that destroys the scalar flux. The term with $E+D$ in the mass-flux budget (65) should, therefore, be interpreted as the term that describes the pressure effects. This is in apparent contradiction with the interpretation of similar terms in the scalar variance budget (58) and in the vertical-velocity variance budget (63), where the terms with $E+D$ describe the dissipation and the combined effect of dissipation and pressure redistribution, respectively. Since the scalar-variance dissipation, the velocity-variance dissipation, the pressure redistribution and the pressure gradient-scalar covariance depend on the flow variables in different ways, it is not easy to describe all the above effects in terms of only two quantities, viz., the rates of lateral entrainment and detrainment (cf. [193, 51]).

A positive outcome of the above analysis of the second-moment budgets is that it suggests an extended formulation for the rates of turbulent entrainment $E$ and detrainment $D$. Recall that the traditional formulation sets $E$ and $D$ proportional to the mass flux $M_{c}$ through the constant fractional entrainment and detrainment rates, $\epsilon$ and $\delta$, respectively (their dimensions is $\mathrm{m}^{-1}$; $\epsilon$ here should not be confused with the TKE dissipation rate). An extended formulation is proposed in [149]. It reads

$$
\begin{aligned}
(E, D) & =M_{c}\left[(\epsilon, \delta)+C_{B} a^{2}(1-a)^{2} \frac{g}{\theta_{r}} \frac{\theta_{u}-\theta_{d}}{\left(M_{c} / \bar{\rho}\right)^{2}}\right] \\
& =M_{c}\left[(\epsilon, \delta)+C_{B} \frac{g}{\theta_{r}} \frac{\theta_{u}-\theta_{d}}{\left(w_{u}-w_{d}\right)^{2}}\right],
\end{aligned}
$$

where $C_{B}$ is a dimensionless constant.

The flow of arguments leading to Eq. (67) is as follows. First, the pressure redistribution term in Eq. (64) and the pressure gradient-scalar covariance term in Eq. (66) are parameterised through Eqs. (12) and (13), respectively, where only the first and the third terms on the r.h.s. are retained. These are return-to-isotropy and the buoyancy parts of the pressure terms that are typically the dominant contributions in convective flows [150, 145]. Next, it is assumed that all pressure relaxation (return-to-isotropy) time scales and 
the dissipation time scales are proportional to each other and to the "master" relaxation time scale $\tau_{m}=e^{-1 / 2} l_{m}, l_{m}$ being the "master" length scale, and that the vertical-velocity variance $\left\langle w^{\prime 2}\right\rangle$ is proportional to the TKE $e$. Then, the above analogies between the mass-flux and the ensemble-mean secondmoment budgets are exploited to infer that $(E+D) / \bar{\rho} \propto a(1-a) / \tau_{m}=$ $a(1-a) e^{1 / 2} / l_{m}$. Finally, using the definition of the convective mass flux (45) and recalling that $\left(w_{u}-w_{d}\right)$ is the mass-flux analogue of $\left\langle w^{\prime 2}\right\rangle^{1 / 2}$ which is assumed to scale on $e$, we obtain $(E, D)=(\epsilon, \delta) M_{c}$ with $(\epsilon, \delta) \propto l_{m}^{-1}$. Then, setting $l_{m}=$ const yields $(\epsilon, \delta)=$ const. It is easy to verify that the second term in brackets on the r.h.s. of Eq. (67) stems from the buoyancy contributions to the pressure terms in the budgets of the vertical-velocity variance and of the scalar flux.

Notice that the traditional formulation for $E$ and $D$, i.e. Eq. (67) without the second term in brackets on the r.h.s., can be obtained from the above reasoning if the simplest Rotta-type formulations (10) and (11) are used instead of (12) and (13), respectively, to parameterise the pressure terms in the second-moment budgets. It should also be mentioned that the above analysis of the second-moment budgets does not allow to discriminate between $E$ and $D$. That is, it provides no guidance as to whether the buoyancy correction term in Eq. (67) should be applied to $E$, to $D$, or to both $E$ and $D$.

A formulation for the fractional entrainment rate $\epsilon$ that is very similar to the second term in brackets on the r.h.s. of Eq. (67) was proposed by Gregory [79] from different physical considerations.

\section{Towards a Unified Description of Boundary-Layer Turbulence and Shallow Convection}

Having discussed the second-order closure and the mass-flux modelling frameworks in some detail, it is appropriate to return to the question raised in the Introduction. That is, whether regime-dependent parameterisation schemes should be developed to describe various types of fluctuating motions, or some unification of different parameterisation frameworks could be achieved. Although a definitive answer to this question does not seem to exist at present, there is a growing interest in unifying various parameterisation ideas (see discussions in $[12,188,146])$. Considering the cumulus parameterisation problem, Arakawa [12] states:

It is rather obvious that for future climate models the scope of the problem must be drastically expanded from "cumulus parameterization" to "unified cloud parameterization" or even to "unified model physics". This is an extremely challenging task, both intellectually and computationally, and the use of multiple approaches is crucial even for a moderate success. 
The tasks of developing a "unified cloud parameterization" and eventually a "unified model physics" are very ambitious. Most NWP and climate models will unlikely enjoy the use of such general parameterisation frameworks for some, perhaps many, years to come. However, a less ambitious task, namely, a unified description of boundary-layer turbulence and shallow convection, seems to be feasible. There are several ways to do so, but it is not a priory clear which way should be preferred. A number of attempts have been made to develop a more unified turbulence-shallow convection parameterisation schemes. They can be classified, rather loosely, into three groups.

Extended mass-flux schemes are built around the top-hat updraughtdowndraught representation of fluctuating quantities. As discussed above, the simplest top-hat mass-flux representation is equivalent to assuming a twodelta-function PDF, where the motions can be either updraughts or downdraughts and the sum of the probabilities of the two admissible states is one. Since the variety of motions is not exhausted by quasi-organised updraughts and downdraughts, the mass-flux equations are extended by adding the "sub-plume scale" motions. These motions are thought to be small-scale and chaotic, so that they can be parameterised on the basis of the secondorder closure ideas the simplest of which is the down-gradient approximation of fluxes. Lappen and Randall [111, 112, 113] developed an extended massflux scheme ADHOC (Assumed-Distribution Higher-Order Closure) that parameterises boundary-layer turbulence and shallow convection in a unified framework. As the heart of the scheme is the two-delta-function mass-flux representation, ADHOC is attractive for describing non-local convective transport. Missing components, namely, parameterisations of the sub-plume scale fluxes, of the pressure terms, and, to some extent, of the dissipation terms, are borrowed from the ensemble-mean second-order modelling framework. An updated version of ADHOC, ADHOC2 [114, 115], includes parameterisations of pressure terms and of momentum fluxes consistent with the mass-flux framework (more specifically, with the assumed spatial distribution based on the two types of idealised coherent structures - plumes and rolls).

Parameterisation schemes where the mass-flux closure ideas and the ensemble-mean second-order closure ideas have roughly equal standing can be labelled as hybrid schemes. These are exemplified by the EDMF (EddyDiffusivity/Mass-Flux) scheme proposed by Soares et al. [181] based on earlier work of Siebesma and Teixeira [177]. In the framework of the EDMF scheme, the vertical flux of a fluctuating quantity $f$ is represented as a sum of two contributions [176], one is assumed to stem from the small-scale chaotic eddies and is described with the eddy-diffusivity down-gradient formulation, and the other is assumed to stem from the convective-layer-scale quasi-organised plumes and is described with the mass-flux formulation. That is, $\left\langle w^{\prime} f^{\prime}\right\rangle=-K_{f} \partial\langle f\rangle / \partial z+\left(M_{u} / \bar{\rho}\right)\left(f_{u}-\langle f\rangle\right)$, where $M_{u}$ is the convective updraught mass flux, $f_{u}$ is the value of $f$ in the updraught, and $\langle f\rangle$ is the value of $f$ averaged over a grid box of a host numerical model. The eddy diffusivity $K_{f}$ is estimated on the basis of the TKE, for which a prognostic equation is 
carried, and of a diagnostic formulation for the turbulence length scale [181]. A simple entraining parcel model is used to determine $M_{u}$ and $f_{u}$. The EDMF scheme operates throughout the convective layer, from the (near vicinity of the) surface up to the top of shallow cumuli, and does not require switching between turbulence and convection schemes (until deep convection is triggered that still requires a separate parameterisation scheme). The scheme is formulated in terms of moist quasi-conservative variables, the liquid water potential temperature $\theta_{l}$ and the total water specific humidity $q_{t}$, and an SGS statistical cloud scheme (section 2.5) is used to predict fractional cloud cover and the amount of cloud condensate.

Some features of the EDMF scheme deserve critical consideration. A parcel model used to determine $M_{u}$ and $f_{u}$ assumes that the updraughts fractional area coverage $a_{u}$ is small as compared to the horizontal grid size of a host atmospheric model. Then, the mass-flux component of the EDMF scheme inherits all shortcomings of the "traditional" mass-flux schemes (section 3). In particular, there is no resolution dependency - the mass-flux component remains active irrespective of the ratio of the horizontal size of numerical grid to the size of the updraught. Furthermore, there is no dependency on the skewness of fluctuating fields - the skewness is always large by virtue of a small $a_{u}$. This is an important difference to the ADHOC scheme which guarantees that $a_{u}$ approaches $1 / 2$ as skewness approaches zero.

Variances $\left\langle f^{\prime 2}\right\rangle$ of scalar quantities in the EDMF scheme are diagnosed on the basis of a truncated scalar-variance equation, see Eqs. (6) and (7), where only the dissipation term and the mean-gradient term are retained, that is $\epsilon_{f}=-\left\langle w^{\prime} f^{\prime}\right\rangle \partial\langle f\rangle / \partial z$. Then, parameterising the dissipation rate through the dissipation time scale, $\epsilon_{f}=\left\langle f^{\prime 2}\right\rangle / \tau_{f}$, and using the above EDMF formulation for the flux $\left\langle w^{\prime} f^{\prime}\right\rangle$, yields the expression for $\left\langle f^{\prime 2}\right\rangle$. Notice that using the mass-flux formulation for the flux and at the same time neglecting the third-order transport term in the scalar-variance equation is not quite consistent. Numerous analyses of observational and LES data (e.g. [119, 151, 148]) indicate the importance of the turbulent transport term $-\frac{1}{2} \partial\left\langle w^{\prime} f^{\prime 2}\right\rangle / \partial z$ in maintaining the scalar variance budget in the well-mixed CBL core, where the mean-gradient term is small. Furthermore, as discussed in sections 2.2 and 3.2 , it is the third-order transport term in the scalar-variance equation that accounts for the non-local transport by skewed convective turbulence. Neglecting this term is inconsistent with the assumption of large skewness (small $a_{u}$ ). Notice also that the formulation for $\left\langle f^{\prime 2}\right\rangle$ neglecting the thirdorder transport term should not allow for the counter-gradient scalar flux (when $\left\langle w^{\prime} f^{\prime}\right\rangle$ and $\partial\langle f\rangle / \partial z$ have the same sign) that is known to often occur in convective flows. A counter-gradient scalar flux would lead to totally spurious negative values of the temperature-variance dissipation and hence of the temperature variance. In the EDMF scheme of Soares et al. [181], this situation is avoided by applying a clipping operation - the mass-flux contribution to the scalar-variance is set to zero whenever it becomes negative. As discussed in section 2.5, variances of $\theta_{l}$ and of $q_{t}$ are the key input parameters 
for statistical parameterisations of fractional cloudiness. They should be accurately predicted. This may not be achieved without an accurate treatment of the third-order transport terms in the scalar-variance equations.

Notwithstanding their shortcomings, the EDMF-like hybrid schemes are attractive for they are simple and computationally efficient. They enjoy growing popularity in atmospheric modelling (e.g. [104, 11]).

Non-local second-order closure schemes represent one more alternative to describe boundary-layer turbulence and shallow convection in a unified framework. In pursuing this aim, a number of schemes based on the ensemble-mean equations for the statistical moments of fluctuating fields have been developed. These range from low-order turbulence closures, where the only prognostic equation is the TKE equation (e.g. [21, 19]), to high-order closures, where transport equations are carried for all second-order and third-order moments involved (e.g. [29, 30, 31]). These schemes proved to do a fair job of describing turbulence and shallow convection. Using moist quasi-conservative variables and well-tuned statistical parameterisations of fractional cloudiness, these schemes appeared to be capable of describing cumuliform and stratiform boundary-layer clouds in a unified framework.

Turbulence closure schemes based on the ensemble-mean equations are often blamed for their inability to describe non-local transport due to quasiorganised convective motions. Both heavily truncated second-order closures and sophisticated high-order closures suffer from this drawback. The incorporation of additional transport equations for third-order and possibly higherorder moments makes the schemes very complex and computationally expensive. The gain in terms of accuracy of their performance is, however, not as tangible as one would expect in the hope that making crude assumptions on the high-order moments would still yield an accurate prediction of loworder moments of interest, viz., of fluxes and variances and of the mean fields. What is most likely to be at fault is the assumption, which is either explicit or implicit in most turbulence closures based on the ensemble-mean equations, that the PDF of fluctuating fields is approximately Gaussian. For example, a third-order closure that makes use of the Millionshchikov hypothesis (quasiGaussian approximation, section 2.2) to parameterise fourth-order moments is fairly sophisticated, and yet it fails to properly account for non-local nature of convective turbulence.

As the analysis in sections 2.2 and 3.2 suggests, the inability of traditional ensemble-mean closures to describe non-local convective transport may well be apparent rather than real. In the second-order modelling framework, one of the key points is the parameterisation of the third-order transport moments in the second-moment equations. It is these terms that are largely responsible for non-local transport properties of convective motions. Recall that the third-order terms are usually parameterised through the simple down-gradient diffusive approximations, or through the use of their own transport equations where the fourth-order moments are taken to be Gaussian. In order to account for non-local transport properties of convection, additional terms should be 
added to the formulations for the third-order moments, namely, the terms dependent on the skewness of fluctuating fields. Such additional terms for the third-order moments in the temperature-flux equation and in the temperaturevariance equation are given by Eqs. (19) and (20), respectively. It must be stressed that Eqs. (19) and (20) are simply the mass-flux Eqs. (49) and (50) recast in terms of the ensemble-mean quantities. Since the mass-flux formulations are advective rather than diffusive in character and are intrinsically non-local [111], their ensemble-mean counterparts should also be able to properly account for non-local convective transport. The skewness of fluctuating fields (may be different for different quantities, e.g. [147, 83]) should be determined from transport equations for the third-order moments, where the fourth-order moments are represented through skewness-dependent formulations (21)-(24) (the generalised Millionshchikov hypothesis) which again are consistent with the mass-flux formulations in the non-Gaussian limit. In some situations, simplified algebraic formulations for skewness may appear to be sufficient [19] (see also chapter 4 of this volume).

Notice that extended non-local second-order closure schemes with skewness-dependent formulations for the third-order transport terms are likely to be more stable numerically as they would not violate realisability in case of large skewness. They are also more consistent with statistical parameterisations of fractional cloudiness many of which utilise a skewed PDF to describe cumuliform clouds (see section 2.5).

The above classification of unified turbulence-shallow convection schemes is rather arbitrary. For example, the "assumed PDF scheme" of Golaz et al. $[70,71]$ can be viewed as an extension of the ADHOC scheme of Lappen and Randall, where a two-delta-function PDF is replaced with a two-Gaussianfunction PDF. Alternatively, it can be viewed as an extended ensemble-mean high-order closure based on a rather flexible two-Gaussian PDF. Generally speaking, any of the three approaches outlined above should yield the same result if parameterisations are formulated and implemented clearly and consistently. Putting it differently, it should not matter much which conceptual framework is used as a basis, i.e. whether a unified scheme is built within the mass-flux modelling framework and the missing components (e.g. parameterisation of the sub-plume scale fluxes) are borrowed from the ensemble-mean framework, or whether it is built within the ensemble-mean modelling framework and the missing components (e.g. parameterisation of the third-order transport) are borrowed from the mass-flux framework. However, a clear and consistent formulation requires certain level of complexity, and that level is likely to be higher than most NWP and climate models can afford. In view of stringent requirements of computational economy, simpler schemes are called for that are based on a (heavily) truncated set of equations for statistical moments of fluctuating fields. Mass-flux schemes (or hybrid schemes) are likely to be preferred for some years to come in situations where non-local transport properties of fluctuating fields is a major concern. In a long-term perspective, however, unified schemes built around the ensemble-mean second-order clo- 
sures seem to be more appealing. The following arguments in favour of this viewpoint can be adduced. Due to a rapid development of computers, the resolution of numerical models of the atmosphere is continuously refined. As the mesh size becomes small, increasingly more quasi-organised flow structures, that are chiefly responsible for non-local transport, are resolved. Then, the focus of SGS parameterisations is shifted towards motions at smaller scales, which are (presumably) more chaotic, and towards other issues, such as the anisotropy of turbulence near the surface and in stably stratified regions of the flow and an accurate parameterisation of pressure redistribution and pressure transport. The second-order closures are attractive for describing these very features.

Now we outline the next step that, in the author's opinion, should be made to go beyond the level of one-equation closure schemes (the level 2.5 schemes in the Mellor-Yamada nomenclature) that have been and still are the draft horses of atmospheric turbulence modelling in NWP, climate studies, and related applications. Closure schemes that presently carry only one prognostic equation, viz., the TKE equation, should be extended to incorporate prognostic equations for the scalar variances. This suggestion is almost trivial as may be inferred from the following arguments. The key to successful modelling of any turbulent flow is an adequate description of the flow energy. In neutrally stratified flows, the kinetic energy of turbulence is a major (or the only) concern. This explains why the one-equation closure schemes have been used to advantage in simulating neutral flows. The situation is essentially different in flows where the density (buoyancy) stratification is different from neutral. In such flows, the turbulence potential energy (TPE) plays an important part along with the TKE. The TKE is spent to work against the gravity and is converted into the TPE in stably stratified flows. In convective flows, the TKE grows at the expense of the TPE. The rate of TKE $\leftrightarrow \mathrm{TPE}$ conversion is represented by the buoyancy-flux term $\beta_{i}\left\langle u_{i}^{\prime} \theta_{v}^{\prime}\right\rangle$ that enters the TKE equation (9) as a source (sink) term. Since the atmospheric flows are virtually never hydrostatically neutral, and the TKE and the TPE in stratified flows are equally important, it is difficult to adduce plausible arguments in favour of one form of energy over the other. Both energies should be treated in a similar way. In the dry atmospheric CBL, the potential temperature is the only thermodynamic variable that affects the distribution of buoyancy. The TPE is proportional to the temperature variance [173] that should be determined from Eq. (6), where the representation of the third-order transport term should account for the non-local character of skewed convective motions. In case of moist atmosphere, the TPE depends on the variances $\left\langle\theta_{l}^{\prime 2}\right\rangle$ and $\left\langle q_{t}^{\prime 2}\right\rangle$ of moist quasi-conservative variables and on their correlation $\left\langle\theta_{l}^{\prime} q_{t}^{\prime}\right\rangle$. A parameterisation of fractional cloudiness is additionally required to determine the buoyancy terms.

Closure schemes for atmospheric applications, that carry transport equations for both the TKE and for the scalar variances, have been developed by e.g. Kenjereš and Hanjalić [100] and Nakanishi and Niino [154]. Curiously, it 
was noticed already by Mellor and Yamada in their classical 1974 paper [142] that the scheme (level 3 in their nomenclature) that carries two prognostic equations, for the TKE and for the potential-temperature variance, is particularly attractive. Three closure schemes of various complexity were applied to simulate a PBL subject to a diurnally varying surface heat flux. The level 3 scheme proved to outperform an algebraic closure scheme. Little was gained if the most complex of the three schemes, that carries transport equations for all second-order moments involved, was used. Thus, the level 3 scheme was found to be the best compromise between physical realism and computational economy. The message does not seem to have been got by the geophysical turbulence-modelling community. Most users of the Mellor-Yamada closures gave preference to the level 2.5 scheme in spite of its obvious shortcomings.

In closing this section, yet another way of representing convection and turbulence in numerical models of the atmosphere should be mentioned. Two- or even three-dimensional models capable of resolving cloud scales are embedded into grid-boxes of coarse-resolution atmospheric models. This way to tackle the sub-grid scale parameterisation problem was unthinkable a decade ago, but a drastically increased computer power has made it possible nowadays. Consideration of this innovative approach called "cloud-resolving convective parameterisation" [74, 75] or "super-parameterisation" [162] is beyond the scope of the present paper.

\section{Conclusions}

Modelling (parameterising) turbulence and shallow convection in the lower troposphere as it is practised in NWP and related applications is discussed. Although turbulence and convection are both unresolved, sub-grid scale motions and a distinction between the two is quite ambiguous, different concepts are typically used to parameterise them in numerical models of the atmosphere. The ensemble-mean second-order closure approach is taken to describe turbulence, deemed to represent quasi-random small-scale motions, whereas the mass-flux closure approach is taken to describe convection, deemed to represent quasi-organised motions of larger scales.

The ensemble-mean second-order closure framework is outlined with the emphasis on the parameterisation of the pressure redistribution and of the third-order transport. A rather lengthy treatment appeared to be necessary to demonstrate how simplified turbulence parameterisation schemes are obtained and what is lost on the way. As we have seen, only a small fraction of what is available nowadays is actually used in applications. This "keep it simple" strategy is justified in view of stringent requirements of computational economy that parameterisation schemes for NWP, climate modelling and similar applications should necessarily meet. Nonetheless, incorporating more of the essential physics into the existing turbulence schemes is highly desirable. 
The mass-flux closure framework is outlined with reference to its simplifying assumptions and to its limits of applicability. The analogies between the second-moment budgets derived in the mass-flux and in the ensemblemean second-order modelling frameworks are analysed. The analysis shows that the two modelling frameworks have very much in common and that the parameterisation ideas developed in one framework can be translated into the language of the other. Further outcome of the analysis is an extended formulation for the rates of turbulent entrainment and detrainment, Eq. (67), the key parameters in the mass-flux convection schemes.

As the artificial separation of processes and scales in numerical models of the atmosphere causes many conceptual and practical problems [12] (the turbulence-convection separation being an example), there is a growing need for a more consistent description of turbulence and shallow non-precipitating convection within a unified parameterisation framework. Several alternative ways to achieve such a description are considered and their pros and cons are discussed. A non-local second-order closure scheme, that carries prognostic equations for both kinetic energy and potential energy of sub-grid scale fluctuating motions and incorporates skewness-dependent formulations for the third-order moments, seems to be an attractive alternative.

In a long-term perspective, deep precipitating convection should also be incorporated into a unified turbulence-convection scheme. This task is very difficult and intellectually challenging, and quick success is by no means guaranteed. Except for very high resolution atmospheric models capable of resolving deep convective motions, separate parameterisation schemes for deep precipitating convection will be used over some, perhaps many, years to come. Then, these schemes should be adjusted to adequately respond to an increasing resolution of host atmospheric models and to work in harmony with improved turbulence-shallow convection schemes. To this end, some restrictive assumptions of deep convection schemes may need to be relaxed, e.g. the assumptions of steady-state and of small fractional area coverage of convective updraughts. Steps forward in this direction are described in [69, 159, 212].

Acknowledgements. The author is grateful to Wolfgang Hillebrandt and Friedrich Kupka for the invitation to prepare this article, and to Peter Bechtold, Michael Buchhold, Vittorio Canuto, Sergey Danilov, Stephan de Roode, Jean-François Geleyn, Andrey Grachev, Erdmann Heise, Martin Köhler, Friedrich Kupka, Cara-Lyn Lappen, Vasily Lykossov, Detlev Majewski, Pedro Miranda, Veniamin Perov, Jean-Marcel Piriou, David Randall, Matthias Raschendorfer, Bodo Ritter, Pier Siebesma, Pedro Soares, Joao Teixera, JunIchi Yano, Sergej Zilitinkevich, and particularly to Evgeni Fedorovich and Vladimir Gryanik for useful discussions and helpful suggestions at various stages of the preparation of this article. 


\section{References}

1. Abdella, K., and N. McFarlane: A new second-order turbulence closure scheme for the planetary boundary layer. J. Atmos. Sci. 54, 1850-1867 (1997)

2. Abdella, K., and N. McFarlane: Reply. J. Atmos. Sci. 56, 3482-3483 (1999)

3. Abdella, K., and A. C. Petersen: Third-order moment closure through the massflux approach. Boundary-Layer Meteorol. 95, 303-318 (2000)

4. Akylas, E., M. Tombrou, D. Lalas, and S. Zilitinkevich: Surface fluxes under shear-free convection. Quart. J. Roy. Meteorol. Soc. 127, 1-15 (2001)

5. André, J. C., G. De Moor, P. Lacarrère, and R. du Vachat: Turbulence approximation for inhomogeneous flows. Part I: The clipping approximation. J. Atmos. Sci. 33, 476-481 (1976)

6. André, J. C., G. De Moor, P. Lacarrère, and R. du Vachat: Turbulence approximation for inhomogeneous flows. Part II: The numerical simulation of a penetrative convection experiment. J. Atmos. Sci. 33, 482-491 (1976)

7. André, J. C., G. De Moor, P. Lacarrère, G. Therry, and R. du Vachat: Modelling the 24-hour evolution of the mean and turbulent structures of the planetary boundary layer. J. Atmos. Sci. 35, 1861-1883 (1978)

8. Andreas, E. L.: Parameterizing scalar transfer over snow and ice: A review. J. Hydrometeor. 3, 417-432 (2002)

9. Andreas, E. L., and B. A. Cash: Convective heat transfer over wintertime leads and polynyas. J. Geophys. Res. 104, 25721-25734 (1999)

10. Andrén, A.: A TKE-dissipation model for the atmospheric boundary layer. Boundary-Layer Meteorol. 56, 207-221 (1991)

11. Angevine, W. M.: An integrated turbulence scheme for boundary layers with shallow cumulus applied to pollutant transport. J. Appl. Meteorol. 44, 1436$1452(2005)$

12. Arakawa, A.: The cumulus parameterization problem: past, present, and future. J. Climate 17, 2493-2525 (2004)

13. Arakawa, A., and W. H. Schubert: Interaction of a cumulus cloud ensemble with the large-scale environment, Part I. J. Atmos. Sci. 31, 674-701 (1974)

14. Bannon, P. R.: Theoretical foundations for models of moist convection. J. Atmos. Sci. 59, 1967-1982 (2002)

15. Bannon, P. R.: Virtualization. J. Atmos. Sci. 64, 1405-1409 (2007)

16. Baumert, H. Z., J. H. Simpson, and J. Sündermann (Eds.): Marine Turbulence. Theories, Observations, and Models. Cambridge Univ. Press, Cambridge, 630 pp (2005)

17. Bechtold, P., E. Bazile, F. Guichard, P. Mascart, and E. Richard: A mass-flux convection scheme for regional and global models. Quart. J. Roy. Meteorol. Soc. 127, 869-886 (2001)

18. Bechtold, P., J.-P. Chaboureau, A. Beljaars, A. K. Betts, M. Köhler, M. Miller, and J.-L. Redelsperger: The simulation of the diurnal cycle of convective precipitation over land in a global model. Quart. J. Roy. Meteorol. Soc. 130, 3119-3137 (2004)

19. Bechtold, P., J. W. M. Cuijpers, P. Mascart, and P. Trouilhet: Modeling of trade wind cumuli with a low-order turbulence model: Toward a unified description of $\mathrm{Cu}$ and Se clouds in meteorological models. J. Atmos. Sci. 52, 455-463 (1995)

20. Bechtold, P., and P. Siebesma: Organization and representation of boundary layer clouds. J. Atmos. Sci. 55, 888-895 (1998) 
21. Bechtold, P., C. Fravalo, and J.-P. Pinty: A model of marine boundary-layer cloudiness for mesoscale applications. J. Atmos. Sci. 49, 1723-1744 (1992)

22. Beljaars, A. C. M.: The parameterization of surface fluxes in large-scale models under free convection. Quart. J. Roy. Meteorol. Soc. 121, 255-270 (1995)

23. Beljaars, A. C. M., A. R. Brown, and N. Wood: A new parametrization of turbulent orographic form drag. Quart. J. Roy. Meteorol. Soc. 130, 1327-1347 (2004)

24. Beljaars, A. C. M., and A. A. M. Holtslag: Flux parameterization over land surfaces for atmospheric models. J. Appl. Meteorol. 30, 327-341 (1991)

25. Betts, A. K.: Non-precipitating cumulus convection and its parameterization. Quart. J. Roy. Meteorol. Soc. 99, 178-196 (1973)

26. Betts, A. K.: A new convective adjustment scheme. Part I: Observational and theoretical basis. Non-precipitating cumulus convection and its parameterization. Quart. J. Roy. Meteorol. Soc. 112, 677-691 (1986)

27. Betts, A. K., and M. J. Miller: A new convective adjustment scheme. Part II: Single column tests using GATE wave, BOMEX, ATEX and arctic air-mass data sets. Quart. J. Roy. Meteorol. Soc. 112, 693-709 (1986)

28. Blackadar, A. K.: The vertical distribution of wind and turbulent exchange in neutral atmosphere. J. Geophys. Res. 67, 3095-3102 (1962)

29. Bougeault, P.: Modeling the trade-wind cumulus boundary-layer. Part I: Testing the ensemble cloud relations against numerical data. J. Atmos. Sci. 38, 2414-2428 (1981)

30. Bougeault, P.: Modeling the trade-wind cumulus boundary-layer. Part II: A high-order one-dimensional model. J. Atmos. Sci. 39, 2429-2439 (1981)

31. Bougeault, P.: Cloud-ensemble relations based on the Gamma probability distribution for the higher-order models of the planetary boundary layer. J. Atmos. Sci. 39, 2691-2700 (1982)

32. Bougeault, P.: A simple parameterization of the large-scale effects of cumulus convection. Mon. Weather Rev. 113, 2108-2121 (1985)

33. Bradley, F., and C. Fairall: A Guide to Making Climate Quality Meteorological and Flux Measurements at Sea. NOAA Technical Memorandum OAR PSD-311, Earth System Research Laboratory, Boulder, Colorado, USA, 109 pp (2006)

34. Brost, R. A., and J. C. Wyngaard: A model study of the stably stratified planetary boundary layer. J. Atmos. Sci. 35, 1427-1440 (1978)

35. Brutsaert, W.: Evaporation into the Atmosphere. D. Reidel, Dordrecht, etc., 299 pp (1982)

36. Bretherton, F. P.: Momentum transport by gravity waves. Quart. J. Roy. Meteorol. Soc. 95, 213-243 (1969)

37. Burchard, H.: Applied Turbulence Modelling in Marine Waters. Springer, Berlin, etc., 215 pp (2002)

38. Businger, J. A.: Reflections on boundary-layer problems of the last 50 years. Boundary-Layer Meteorol. 4, 323-326 (1973)

39. Businger, J. A.: A note on free convection. Boundary-Layer Meteorol. 116, 161-173 (2005)

40. Bushell, A. C., D. R. Wilson, and D. Gregory: A description of cloud production by non-uniformly distributed processes. Quart. J. Roy. Meteorol. Soc. 129, $1435-1455(2003)$

41. Canuto, V. M., and M. Dubovikov: Stellar turbulent convection. I. Theory. Astrophys. J. 493, 834-847 (1998) 
42. Canuto, V. M., Y. Cheng, and A. Howard: New third-order moments for the convective boundary layer. J. Atmos. Sci. 58, 1169-1172 (2001)

43. Canuto, V. M., F. Minotti, C. Ronchi, R. M. Ypma, and O. Zeman: Secondorder closure PBL model with new third-order moments: comparison with LES data. J. Atmos. Sci. 51, 1605-1618 (1994)

44. Cheng, Y., and V. M. Canuto: Stably stratified shear turbulence: A new model for the energy dissipation length scale. J. Atmos. Sci. 51, 2384-2396 (1994)

45. Chou, P.-Y.: On velocity correlation and the solution of the equation of turbulent fluctuation. Quart. Appl. Math. 3, 38-54 (1945)

46. Craft, T. J., N. Z. Ince, and B. E. Launder: Recent developments in secondmoment closure for buoyancy-affected flows. Dyn. Atmos. Oceans 23, 99-114 (1996)

47. Cuijpers, J. W. M., and P. Bechtold: A simple parameterization of cloud water related variables for use in boundary layer models. J. Atmos. Sci. 52, 2486-2490 (1995)

48. Cullen, M. J. P.: The unified forecast/climate model. Meteor. Mag. 122, 81-94 (1993)

49. Daly, B. J., and F. H. Harlow: Transport equations in turbulence. Phys. Fluids 13, 2634-2649 (1970)

50. Dakos, T., and M. M. Gibson, 1987: On modelling the pressure terms of the scalar flux equations. In: Turbulent Shear Flows 5, ed by F. Durst et al., (Springer, Berlin, 1987) pp 7-18

51. de Roode, S. R., and C. S. Bretherton: Mass-flux budgets of shallow cumulus clouds. J. Atmos. Sci. 60, 137-151 (2003)

52. de Roode, S. R., P. G. Duynkerke, and A. P. Siebesma: Analogies between massflux and Reynolds-averaged equations. J. Atmos. Sci. 57, 1585-1598 (2000)

53. Deardorff, J. W.: Preliminary results from numerical integrations of the unstable planetary boundary layer. J. Atmos. Sci. 27, 1209-1211 (1970)

54. Deardorff, J. W.: Convective velocity and temperature scales for the unstable planetary boundary layer and for Rayleigh convection. J. Atmos. Sci. 27, 12111213 (1970)

55. Deardorff, J. W.: The use of subgrid transport equations in a three-dimensional model of atmospheric turbulence. J. Fluids Eng. 95, 429-438 (1973)

56. Deardorff, J. W.: Usefulness of liquid-water potential temperature in a shallowcloud model. J. Appl. Meteorol. 15, 98-102 (1976)

57. du Vachat, R.: Realizability inequalities in turbulent flows. Phys. Fluids 20, 551-556 (1977)

58. Duynkerke, P. G.: Application of the $E-\epsilon$ turbulence closure model to the neutral and stable atmospheric boundary layer. J. Atmos. Sci. 45, 865-880 (1988)

59. Emanuel, K. A.: A scheme for representing cumulus convection in large-scale models. J. Atmos. Sci. 48, 2313-2335 (2001)

60. Emanuel, K. A.: Atmospheric Convection. Oxford Univ. Press, Oxford, 580 pp (1994)

61. Fedorovich, E., R. Rotunno, and B. Stevens (Eds.): Atmospheric Turbulence and Mesoscale Meteorology. Cambridge Univ. Press, Cambridge, 280 pp (2004)

62. Finnigan, J. J.: A note on wave-turbulence interaction and the possibility of scaling the very stable boundary layer. Boundary-Layer Meteorol. 90, 529-539 (1999) 
63. Fleagle, R. G.. and J. A. Businger: An Introduction to Atmospheric Physics. Academic Press, New York, 346 pp (1963)

64. Foken, T.: 50 years of the Monin-Obukhov similarity theory. Boundary-Layer Meteorol. 119, 431-447 (2006)

65. Frank, W. M.: The cumulus parameterization problem. Mon. Weather Rev. 111, 1859-1871 (1983)

66. Galperin, B., and S. A. Orszag (Eds.): Large Eddy Simulation of Complex Engineering and Geophysical Flows. Cambridge Univ. Press, Cambridge, 622 pp (1993)

67. Garratt, J. R.: The Atmospheric Boundary Layer. Cambridge Univ. Press, Cambridge, 316 pp (1992)

68. Geernaert, G. L. (Ed.): Air-Sea Exchange: Physics, Chemistry and Dynamics. Kluwer Acad. Publ., Dordrecht, etc., 578 pp (1999)

69. Gerard, L., and J.-F. Geleyn: Evolution of a subgrid convection parameterization in a limited-area model with increasing resolution. Quart. J. Roy. Meteorol. Soc. 131, 2293-2312 (2005)

70. Golaz, J.-C., V. E. Larson, and W. R. Cotton: A PDF-based model for boundary layer clouds. Part I: Method and model description. J. Atmos. Sci. 59, 35403551 (2002)

71. Golaz, J.-C., V. E. Larson, and W. R. Cotton: A PDF-based model for boundary layer clouds. Part II: Model results. J. Atmos. Sci. 59, 3552-3571 (2002)

72. Golitsyn, G. S., and A. A. Grachev: Velocities and heat and mass transfer during convection in a two-component medium. Doklady Acad. Nauk SSSR 255, 548-552 (1980)

73. Golitsyn, G. S., and A. A. Grachev: Free convection of multi-component media and parameterization of air-sea interaction at light winds. Ocean-Air Interactions 1, 57-78 (1986)

74. Grabowski, W. W.: MJO-like coherent structures: sensitivity simulations using cloud-resolving convection parameterization (CRCP). J. Atmos. Sci. 60, 847876 (2003)

75. Grabowski, W. W., and P. K. Smolarkiewicz: CRCP: a cloud resolving convection parameterization for modeling the tropical convective atmosphere. Physica D 133, 171-178 (1999)

76. Grachev, A. A., E. L. Andreas, Ch. W. Fairall, P. S. Guest, and P. O. G. Persson: Turbulent measurements in the stable atmospheric boundary layer during SHEBA: ten years after. Acta Geophys. 56, 142-166 (2008)

77. Grachev, A. A., C. W. Fairall, P. O. G. Persson, E. L. Andreas, and P. S. Guest: Stable boundary-layer scaling regimes: the SHEBA data. Boundary-Layer Meteorol. 116, 201-235 (2005)

78. Grachev, A. A., and G. N. Panin: Parameterization of the sensible and latent heat fluxes above the water surface in calm weather under natural conditions. Izvestija AN SSSR. Fizika Atmosfery i Okeana 20, 364-371 (1984)

79. Gregory, D.: Estimation of entrainment rate in simple models of convective clouds. Quart. J. Roy. Meteorol. Soc. 127, 53-72 (2001)

80. Gregory, D., R. Kershaw, and P. M. Inness: Parametrization of momentum transport by convection. II: Tests in single-column and general circulation models. Quart. J. Roy. Meteorol. Soc. 123, 1153-1183 (1997)

81. Gregory, D., D. Wilson, and A. Bushell: Insights into cloud parametrization provided by a prognostic approach. Quart. J. Roy. Meteorol. Soc. 128, 14851504 (2002) 
82. Gregory, D., and P. R. Rowntree: A mass flux convection scheme with representation of cloud ensemble characteristics and stability-dependent closure. Mon. Weather Rev. 118, 1483-1506 (1990)

83. Gryanik, V. M., and J. Hartmann: A turbulence closure for the convective boundary layer based on a two-scale mass-flux approach. J. Atmos. Sci. 59, 2729-2744 (2002)

84. Gryanik, V. M., J. Hartmann, S. Raasch, and M. Schröter: A refinement of the Millionshchikov quasi-normality hypothesis for convective boundary layer turbulence. J. Atmos. Sci. 62, 2632-2638 (2005)

85. Hallbäck, M., D. S. Henningson, A. V. Johansson, and P. H. Alfredsson (Eds.): Turbulence and Transition Modelling. Kluwer Acad. Publ., Dordrecht, etc., 369 pp (1996)

86. Hanjalić, K., and B. E. Launder: A Reynolds stress model of turbulence and its application to thin shear flows. J. Fluid Mech. 52, 609-638 (1972)

87. Hassid, S., and B. Galperin: Modeling rotating flows with neutral and unstable stratification. J. Geophys. Res. 99, 12,533-12,548 (1994)

88. Högström, U.: Non-dimensional wind and temperature profiles in the atmospheric surface layer: A re-evaluation. Boundary-Layer Meteorol. 42, 55-78 (1988)

89. Högström, U.: Review of some basic characteristics of the atmospheric surface layer. Boundary-Layer Meteorol. 78, 215-246 (1996)

90. Holton, J. R.: A one-dimensional cumulus model including pressure perturbations. Mon. Weather Rev. 101, 201-205 (1973)

91. Houze, R. A.: Cloud Dynamics. Academic Press, San Diego, etc., 573 pp (1993)

92. Holtslag, A. A. M., and B. Boville: Local versus nonlocal boundary-layer diffusion in a global climate model. J. Climate 6, 1825-1842 (1993)

93. IFS Documentation, Cycle 28r1. Available from the web site of ECMWF, http://www.ecmwf.int (2004)

94. Jones, W. P., and P. Musogne: Modelling of scalar transport in homogeneous turbulent flows. In: Proc. of the 4th Turbulent Shear Flows Symposium, Karlsruhe, Germany, pp 17.18-17.24 (1983)

95. Kaimal, J. C., and J. J. Finnigan: Atmospheric Boundary Layer Flows. Their Structure and Measurements. Oxford Univ. Press, New York, 289 pp (1994)

96. Kain, J. S.: The Kain-Fritsch convection parameterization: an update. J. Appl. Meteorol. 43, 170-181 (2004)

97. Kain, J. S., and J. M. Fritsch: A one-dimensional entraining/detraining plume model and its application in convective parameterization. J. Atmos. Sci. 47, 2784-2802 (1990)

98. Kain, J. S., and J. M. Fritsch, 1993: Convective parameterization for mesoscale models: the Kain-Fritsch scheme. In: The Representation of Cumulus Convection in Numerical Models, Meteorol. Monogr. No. 24, Amer. Meteor. Soc., pp 165-170 (1993)

99. Kantha, L. H., and C. A. Clayson: Small Scale Processes in Geophysical Fluid Flows. Academic Press, San Diego, etc., 888 pp (2000)

100. Kenjereš, S., and K. Hanjalić: Combined effects of terrain orography and thermal stratification on pollutant dispersion in a town valley: a T-RANS simulation. J. Turbulence 3, 1-25, doi:10.1088/1468-5248/3/1/026 (2002)

101. Kenjereš, S., and K. Hanjalić: Numerical simulation of magnetic control of heat transfer in thermal convection. Int. J. Heat Fluid Fl. 16, 429-439 (2004) 
102. Kershaw, R., and D. Gregory: Parametrization of momentum transport by convection. I: Theory and cloud modelling results. Quart. J. Roy. Meteorol. Soc. 123, 1133-1151 (1997)

103. Kitaigorodskii, S. A.: The Physics of Air-Sea Interaction. Gidrometeoizdat, Leningrad, 284 pp (1970) (English translation by A. Baruch, Israel Program for Scientific Translation, Jerusalem, 1973, 273 pp.)

104. Köhler, M: Improved prediction of boundary layer clouds. ECMWF Newsletter, No. 104, 18-22 (2005)

105. Kolmogorov, A. N.: Local structure of turbulence in an incompressible fluid at very high Reynolds numbers. Doklady Acad. Nauk SSSR 30, 299-303 (1941)

106. Komen, G. J., L. Cavalery, M. Donelan, K. Hasselmann, S. Hasselmann, and P. A. E. M. Janssen: Dynamics and Modelling of Ocean Waves. Cambridge Univ. Press, Cambridge, 532 pp (1994)

107. Kuo, H. L.: On formation and intensification of tropical cyclones through latent heat release by cumulus convection. J. Atmos. Sci. 22, 40-63 (1965)

108. Kuo, H. L.: Further studies of the parameterization of the influence of cumulus convection on large-scale flow. J. Atmos. Sci. 31, 1232-1240 (1974)

109. Kupka, F, and F. J. Robinson: On the effects of coherent structures on higher order moments in models of solar and stellar surface convection. Mon. Not. R. Astron. Soc. 374, 305-322 (2007)

110. Lange, H.-J.: Die Physik des Wetters und des Klimas. Ein Grundkurs zur Theorie des Systems Atmosphäre. Dietrich Reimer Verlag, Berlin, 625 pp (2002) (in German)

111. Lappen, C.-L., and D. A. Randall: Toward a unified parameterization of the boundary layer and moist convection. Part I: A new type of mass-flux model. J. Atmos. Sci. 58, 2021-2036 (2001)

112. Lappen, C.-L., and D. A. Randall: Toward a unified parameterization of the boundary layer and moist convection. Part II: Lateral mass exchanges and subplume-scale fluxes. J. Atmos. Sci. 58, 2037-2051 (2001)

113. Lappen, C.-L., and D. A. Randall: Toward a unified parameterization of the boundary layer and moist convection. Part III: Simulations of clear and cloudy convection. J. Atmos. Sci. 58, 2052-2072 (2001)

114. Lappen, C.-L., and D. A. Randall: Using idealized coherent structures to parameterize momentum fluxes in a PBL mass-flux model. J. Atmos. Sci. 62, 2829-2846 (2005)

115. Lappen, C.-L., and D. A. Randall: Parameterization of pressure perturbations in a PBL mass-flux model. J. Atmos. Sci. 63, 1726-1751 (2006)

116. Larson, V. E: Prognostic equations for cloud fraction and liquid water, and their relation to filtered density functions. J. Atmos. Sci. 61, 338-351 (2004)

117. Larson, V. E, and J.-C. Golaz: Using probability density functions to derive consistent closure relationships among higher-order moments. Mon. Weather Rev. 133, 1023-1042 (2005)

118. Launder, B. E., G. J. Reece, and W. Rodi: Progress in the development of a Reynolds stress turbulence closure. J. Fluid Mech. 68, 537-566 (1975)

119. Lenschow, D. H., J. C. Wyngaard, and W. T. Pennell: Mean-field and secondmoment budgets in a baroclinic, convective boundary layer. J. Atmos. Sci. 37, 1313-1326 (1980)

120. Lenschow, D. H., J. Mann, and L. Kristensen: How long is long enough when measuring fluxes and other turbulence statistics? J. Atmos. Oceanic Technol. 11, 661-673 (1994) 
121. Lesieur, M.: Turbulence in Fluids. Kluwer Acad. Publ., Dordrecht, etc., 515 pp (1997)

122. Lewellen, D. C., and W. S. Lewellen: Buoyancy flux modeling for cloudy boundary layers. J. Atmos. Sci. 61, 1147-1160 (2004)

123. Lewellen, W. C., and S. Yoh: Binormal model of ensemble partial cloudiness. J. Atmos. Sci. 50, 1228-1237 (1993)

124. Lilly, D. K.: The representation of small-scale turbulence in numerical simulation experiments. In: Proc. of the IBM Scientific Computing Symposium on Environmental Sciences, Yorktown Heights, New York, Thomas J. Watson Research Center, IBM, pp 195-210 (1967)

125. Lilly, D. K.: Models of cloud-topped mixed layers under a strong inversion. Quart. J. Roy. Meteorol. Soc. 94, 292-309 (1968)

126. Lock, A. P.: The parameterization of entrainment in cloudy boundary layers. Quart. J. Roy. Meteorol. Soc. 124, 2729-2753 (1998)

127. Lock, A. P.: The numerical representation of entrainment in parameterizations of boundary layer turbulent mixing. Mon. Weather Rev. 129, 3187-3199 (2001)

128. Lock, A. P., A. R. Brown, M. R. Bush, G. M. Martin, and R. N. Smith: A new boundary layer mixing scheme. Part I: Scheme description and single-column model tests. Mon. Weather Rev. 128, 3187-3199 (2000)

129. Losch, M.: On the validity of the Millionshchikov quasi-normality hypothesis for open-ocean deep convection. Geophys. Res. Lett. 31, L23301, doi:10.1029/2004GL021412 (2004)

130. Lott, F. and M. J. Miller: A new subgrid-scale orographic drag parametrization: its formulation and testing. Quart. J. Roy. Meteorol. Soc. 123, 101-127 (1997)

131. Lumley, J. L.: Computational modeling of turbulent flows. Adv. Appl. Mech. 18, 123-176 (1978)

132. Lumley, J. L., and H. Panofsky: The Structure of Atmospheric Turbulence. John Wiley and Sons, New York, 239 pp (1964)

133. Mahrt, L.: Stratified atmospheric boundary layers and breakdown of models. J. Theor. Comp. Flud Dyn. 11, 263-279 (1998)

134. Mahrt, L.: Stratified atmospheric boundary layers. Boundary-Layer Meteorol. 90, 375-396 (1999)

135. Mahrt, L., J. Sun, W. Blumen, T. Delany, and S. Oncley: Nocturnal boundarylayer regimes. Boundary-Layer Meteorol. 88, 375-396 (1998)

136. Majewski, D., D. Liermann, P. Prohl, B. Ritter, M. Buchhold, T. Hanisch, G. Paul, W. Wergen, and J. Baumgardner: Icosahedral-hexagonal gridpoint model GME: description and high-resolution tests. Mon. Weather Rev. 130, 319-338 (2002)

137. Malhi, Y.: The behaviour of the roughness length for temperature over heterogeneous surfaces. Quart. J. Roy. Meteorol. Soc. 122, 1095-1125 (1996)

138. Marshall, J., and F. Schott: Open-ocean convection: Observations, theory, and models. Rev. Geophys. 37, 1-64 (1999)

139. Martin, G. M., M. R. Bush, A. R. Brown, A. P. Lock, and R. N. Smith: A new boundary layer mixing scheme. Part II: Tests in climate and mesoscale models. Mon. Weather Rev. 128, 3200-3217 (2000)

140. McFarlane, N. A.: The effect of orographically excited gravity wave drag on the circulation of the lower stratosphere and troposphere. J. Atmos. Sci. 44, 1775-1800 (1987)

141. Mellor, G. L.: The Gaussian cloud model relations. J. Atmos. Sci. 34, 356-358 [Corrigendum 1483-1484] (1977) 
142. Mellor, G. L., and T. Yamada: A hierarchy of turbulence closure models for planetary boundary layers. J. Atmos. Sci. 31, 1791-1806 (1974)

143. Mellor, G. L., and T. Yamada: Development of a turbulence closure model for geophysical fluid problems. Rev. Geophys. 20, 851-875 (1982)

144. Millionshchikov, M. D.: On the theory of homogeneous isotropic turbulence. Doklady Acad. Nauk SSSR 32, 611-614 (1941)

145. Mironov, D. V.: Pressure-potential-temperature covariance in convection with rotation. Quart. J. Roy. Meteorol. Soc. 127, 89-100 (2001)

146. Mironov, D., and C. Jones: Summary of the working group discussion on the representation of convection in high resolution numerical models. In: Proc. of the HIRLAM/NetFAM Workshop on Convection and Clouds, 24-26 January 2005, Tartu, Estonia, pp 113-116 (2005)

147. Mironov, D. V., V. M. Gryanik, V. N. Lykossov, and S. S. Zilitinkevich: Comments on "A New Second-Order Turbulence Closure Scheme for the Planetary Boundary Layer" by K. Abdella and N. McFarlane. J. Atmos. Sci. 56, 34783481 (1999)

148. Mironov, D. V., V. M. Gryanik, C.-H. Moeng, D. J. Olbers, and T. H. Warncke: Vertical turbulence structure and second-moment budgets in convection with rotation: a large-eddy simulation study. Quart. J. Roy. Meteorol. Soc. 126, 477-515 (2000)

149. Mironov, D., and B. Ritter: Parameterization of convection in the global NWP system GME of the German Weather Service. In: Proc. of the HIRLAM/NetFAM Workshop on Convection and Clouds, 24-26 January 2005, Tartu, Estonia, pp 68-72 (2005)

150. Moeng, C.-H., and J. C. Wyngaard: An analysis of closures for pressure-scalar covariances in the convective boundary layer. J. Atmos. Sci. 43, 2499-2513 (1986)

151. Moeng, C.-H., and J. C. Wyngaard: Evaluation of turbulent transport and dissipation closure in second order modelling. J. Atmos. Sci. 46, 2311-2330 (1989)

152. Monin, A. S., and A. M. Obukhov: Basic laws of turbulent mixing in the atmospheric surface layer. Trudy Geofiz. Inst. Akad. Nauk SSSR No. 24 (151), 163-187 (1954)

153. Monin, A. S., and A. M. Yaglom: Statistical Fluid Mechanics. Vol. 1. MIT Press, Cambridge, Massachusetts, 769 pp (1971)

154. Nakanishi, M., and H. Niino: An improved Mellor-Yamada level-3 model with condensation physics: its design and verification. Boundary-Layer Meteorol. 112, 1-31 (2004)

155. Nurser, A. J. G.: A Review of Models and Observations of the Oceanic Mixed Layer. Southampton Oceanographic Centre, Internal document No. 14, Southampton, U.K., 247 pp (1996)

156. Obukhov, A. M.: Turbulence in an atmosphere with a non-uniform temperature. Trudy Inst. Teor. Geofiz. Akad. Nauk SSSR 1, 95-115 (1946) (English translation: Boundary-Layer Meteorol. 2, 7-29, 1971)

157. Otić, I., G. Grötzbach, and M. Wörner: Analysis and modelling of the temperature variance equation in turbulent natural convection for low-Prandtl-number fluids. J. Fluid Mech. 525, 237-261 (2005)

158. Perov, V., and S. Zilitinkevich: Application of an extended similarity theory for the stably stratified atmospheric surface layer to the HIRLAM. HIRLAM Newsletter, No. 35, 137-142 (2000) 
159. Piriou, J.-M., J.-L. Redelsperger, J.-F. Geleyn, J.-P. Lafore, and F. Guichard: An approach for convective parameterization with memory: separating microphysics and transport in grid-scale equations. J. Atmos. Sci. 64, 4127-4139 (2007)

160. Plate, E. J., E. E. Fedorovich, D. X. Viegas, and J. C. Wyngaard (Eds.): Buoyant Convection in Geophysical Flows. Kluwer Acad. Publ., Dordrecht, etc., 504 pp (1998)

161. Pope, S. B.: Turbulent Flows. Cambridge Univ. Press, Cambridge, 771 pp (2000)

162. Randall, D., M. Khairoutdinov, A. Arakawa, and W. Grabowski: Breaking the cloud parameterization deadlock. Bull. Amer. Met. Soc. 84, 1547-1564 (2003)

163. Randall, D. A., Q. Shao, and C.-H. Moeng: A second-order bulk boundary-layer model. J. Atmos. Sci. 49, 1903-1923 (1992)

164. Raschendorfer, M.: Special topic: The new turbulence parameterization of LM. Quarterly Report of the Operational NWP-Models of the Deutscher Wetterdienst, No. 19, 3-12 (1999)

165. Raschendorfer, M.: The new turbulence parameterization of LM. COSMO Newsletter, No. 1, 89-97 (2001) (available from www.cosmo-model.org)

166. Raupach, M. R.: Drag and drag partition on rough surfaces. Boundary-Layer Meteorol. 60, 375-395 (1992)

167. Ristorcelli, J. R., J. L. Lumley, and R. Abid: A rapid-pressure covariance representation consistent with the Taylor-Proudman theorem materially frame indifferent in the two-dimensional limit. J. Fluid Mech. 292, 111-152 (1995)

168. Rodi, W.: Examples of calculation methods for flow and mixing in stratified fluids. J. Geophys. Res. 92, 5305-5328 (1987)

169. Rotta, J. C.: Statistische Theorie nichthomogener Turbulenz. 1. Zs. Phys. 129, 547-572 (1951)

170. Sander, J.: Dynamical equations and turbulent closures in geophysics. Continuum Mech. Therm. 10, 1-28 (1998)

171. Schumann, U.: Realizability of Reynolds-stress turbulence models. Phys. Fluids 20, 721-725 (1977)

172. Schumann, U.: Minimum friction velocity and heat transfer in the rough surface layer of a convective boundary layer. Boundary-Layer Meteorol. 44, 311-326 (1988)

173. Schumann, U., and T. Gerz: Turbulent mixing in stably stratified shear flows. J. Appl. Meteorol. 34, 33-48 (1995)

174. Shih, T.-H.: Constitutive relations and realizability of single-point turbulence closures. In: Turbulence and Transition Modelling, ed by M. Hallbäck, D. S. Henningson, A. V. Johansson, and P. H. Alfredsson, (Kluwer Acad. Publ., Dordrecht, etc., 1996) pp 155-192

175. Siebesma, A. P., 1998: Shallow cumulus convection. In: Buoyant Convection in Geophysical Flows, ed by E. Plate et al., (Kluwer Acad. Publ., Dordrecht, etc., 1998) pp 441-486

176. Siebesma, A. P., and J. W. M. Cuijpers: Evaluation of parametric assumptions for shallow cumulus convection. J. Atmos. Sci. 55, 650-666 (1995)

177. Siebesma, A. P. and J. Teixeira: An advection-diffusion scheme for the convective boundary layer: Description and $1 \mathrm{D}$ results. In: Proc. of the 14 th $A M S$ Symposium on Boundary Layers and Turbulence, (Aspen, CO), American Meteorol. Soc, Boston, USA, pp 133-136 (2000) 
178. Simpson, J., R. H. Simpson, D. A. Andrews, and M. A. Eaton: Experimental cumulus dynamics. Rev. Geophys. 3, 387-431 (1965)

179. Simpson, J., and V. Wiggert: Models of precipitating cumulus towers. Mon. Weather Rev. 97, 471-489 (1969)

180. Smith, R. K.: The role of cumulus convection in hurricanes and its representation in hurricane models. Rev. Geophys. 38, 465-489 (2000)

181. Soares, P. M. M., P. M. A. Miranda, A. P. Siebesma, and J. Teixeira: An eddydiffusivity/mass-flux parameterization for dry and shallow cumulus convection. Quart. J. Roy. Meteorol. Soc. 130, 3365-3383 (2004)

182. Sommeria, G., and J. W. Deardorff: Subgrid-scale condensation in models of nonprecipitating clouds. J. Atmos. Sci. 34, 344-355 (1977)

183. Soomere, T., and S. S. Zilitinkevich: Supplement to 'Third-order transport due to internal waves and non-local turbulence in the stably stratified surface layer'. Quart. J. Roy. Meteorol. Soc. 128, 1029-1031 (2002)

184. Sorbjan, Z.: Structure of the Atmospheric Boundary Layer. Prentice Hall, Englewood Cliffs, New Jersey 07632, 317 pp (1989)

185. Speziale, C. G.: Modeling the pressure gradient-velocity correlation of turbulence. Phys. Fluids 28, 69-71 (1985)

186. Stensrud, D. J.: Parameterization Schemes: Keys to Understanding Numerical Weather Prediction Models. Cambridge Univ. Press, Cambridge, 478 pp (2007)

187. Steppeler, J., G. Doms, U. Schättler, H. W. Bitzer, A. Gassmann, U. Damrath, and G. Gregoric, 2003: Meso-gamma scale forecasts using the non-hydrostatic model LM. Meteorol. Atmos. Phys. 82, 75-96 (2003)

188. Stevens, B.: Atmospheric moist convection. Ann. Rev. Earth Planet. Sci. 33, 605-643 (2005)

189. Stull, R. B.: A convective transport theory for surface fluxes. J. Atmos. Sci. 51, 3-22 (1994)

190. Sukoriansky, S., and B. Galperin: A spectral closure model for turbulent flows with stable stratification. In: Marine Turbulence. Theories, Observations, and Models, ed by H. Z. Baumert, J. H. Simpson, and J. Sündermann, (Cambridge Univ. Press, Cambridge, 2005) pp 53-65

191. Sukoriansky, S., B. Galperin, and V. Perov: Application of a new spectral theory of stably stratified turbulence to the atmospheric boundary layer over sea ice. Boundary-Layer Meteorol. 117, 231-257 (2005)

192. Sukoriansky, S., B. Galperin, and I. Staroselsky: A quasinormal scale elimination model of turbulent flows with stable stratification. Phys. Fluids 17, 085107-085107-28, doi:10.1063/1.2009010 (2005)

193. Swann, H.: Evaluation of the mass-flux approach to parametrizing deep convection. Quart. J. Roy. Meteorol. Soc. 127, 1239-1260 (2001)

194. Sykes, R. I., D. S. Henn, and W. S. Lewellen: Surface-layer description under free-convection conditions. Quart. J. Roy. Meteorol. Soc. 119, 409-421 (1993)

195. Tennekes, H., and J. L. Lumley: A First Course in Turbulence. MIT Press, Cambridge, Massachusetts, 300 pp (1972)

196. Tiedtke, M.: The Parameterization of Moist Processes. Part 2: Parameterization of Cumulus Convection. Meteorological Training Course, Lecture Series, European Centre for Medium-Range Weather Forecasts, Reading, U.K., 78 pp (1988)

197. Tiedtke, M.: A comprehensive mass flux scheme for cumulus parameterization in large-scale models. Mon. Weather Rev. 117, 1779-1800 (1989) 
198. Tiedtke, M.: Representation of clouds in large-scale models. Mon. Weather Rev. 121, 3040-3061 (1993)

199. Tompkins, A. M.: A prognostic parameterization for the subgrid-scale variability of water vapor and clouds in large-scale models and its use to diagnose cloud cover. J. Atmos. Sci. 59, 1917-1942 (2002)

200. Tompkins, A. M.: The parameterization of cloud cover. Technical Memorandum, European Centre for Medium-Range Weather Forecasts, Reading, U.K., $23 \mathrm{pp}(2005)$

201. Troen, I., and L. Mahrt: A simple model of the atmospheric boundary layer; sensitivity to surface evaporation. Boundary-Layer Meteorol. 37, 129-148 (1986)

202. Umlauf, L., and H. Burchard: A generic length-scale equation for geophysical turbulence models. J. Marine Res. 61, 235-265 (2003)

203. Umlauf, L., and H. Burchard: Second-order turbulence closure models for geophysical boundary layers. A review of recent work. Cont. Shelf Res. 25, 795-827 (2005)

204. Umlauf, L., H. Burchard, and K. Hutter: Extending the $k-\omega$ turbulence model towards oceanic applications. Ocean Modelling 5, 195-218 (2003)

205. Unden, P., and Co-authors: HIRLAM-5 Scientific Documentation. HIRLAM Report, Swedish Meteorological and Hydrological Institute, 144 pp (2002)

206. Wilcox, D. C.: Reassessment of the scale-determining equation for advanced turbulence models. AIAA J. 26, 1299-1310 (1988)

207. Wilson, D., and D. Gregory: The behaviour of large-scale model cloud schemes under idealized forcing scenarios. Quart. J. Roy. Meteorol. Soc. 129, 967-986 (2003)

208. Wu, X., and M. Yanai: Effects of vertical wind shear on the cumulus transport of momentum: Observations and parameterizations. J. Atmos. Sci. 51, 16401660 (1994)

209. Wyngaard, J.: Atmospheric turbulence. Ann. Rev. Fluid Mech. 24, 205-233 (1992)

210. Wyngaard, J.: A physical mechanism for the asymmetry in top-down and bottom-up diffusion. J. Atmos. Sci. 44, 1083-1087 (1987)

211. Wyngaard, J.: Toward numerical modeling in the "Terra Incognita". J. Atmos. Sci. 61, 1816-1826 (2004)

212. Yano, J.-I., and J.-M. Piriou: Deduction of subgrid-scale physical processes from an explicit model to massflux parameterization. Submitted to J. Atmos. Sci. (2008)

213. Yau, M. K.: Perturbation pressure and cumulus convection. J. Atmos. Sci. 36, 690-694 (1979)

214. Zeierman, S., and M. Wolfstein: Turbulent time scale for turbulent-flow calculations. AIAA J. 24, 1606-1610 (1986)

215. Zeman, O.: Progress in the modelling of planetary boundary layers. Ann. Rev. Fluid Mech. 13, 253-272 (1981)

216. Zeman, O., and J. L. Lumley: Modeling buoyancy driven mixed layers. J. Atmos. Sci. 33, 1974-1988 (1976)

217. Zeman, O., and H. Tennekes: Parameterization of the turbulent energy budget at the top of the daytime atmospheric boundary layer. J. Atmos. Sci. 34, 111123 (1977)

218. Zilitinkevich, S. S.: Dynamics of the Atmospheric Boundary Layer. Gidrometeoizdat, Leningrad, 292 pp (1970) (in Russian) 
219. Zilitinkevich, S.: Third-order transport due to internal waves and non-local turbulence in the stably stratified surface layer. Quart. J. Roy. Meteorol. Soc. 128, 913-925 (2002)

220. Zilitinkevich, S., A. Baklanov, J. Rost, A.-S. Smedman, V. Lykosov, and P. Calanca: Diagnostic and prognostic equations for the depth of the stably stratified Ekman boundary layer. Quart. J. Roy. Meteorol. Soc. 128, 25-46 (2002)

221. Zilitinkevich, S., and P. Calanca: An extended similarity-theory for the stably stratified atmospheric surface layer. Quart. J. Roy. Meteorol. Soc. 126, 19131923 (2000)

222. Zilitinkevich, S. S., and I. N. Esau: Resistance and heat-transfer laws for neutral and stable planetary boundary layers: Old theory advanced and reevaluated. Quart. J. Roy. Meteorol. Soc. 131, 1863-1892 (2005)

223. Zilitinkevich, S. S.; J. C. R. Hunt, I. N. Esau, A. A. Grachev, D. P. Lalas, E. Akylas, M. Tombrou, C. W. Fairall, H. J. S. Fernando, A. A. Baklanov, and S. M. Joffrer: The influence of large convective eddies on the surface-layer turbulence. Quart. J. Roy. Meteorol. Soc. 132, 1423-1456 (2006)

224. Zilitinkevich, S., P.-E. Johansson, D. V. Mironov, and A. Baklanov: A similarity-theory model for wind profile and resistance law in stably stratified planetary boundary layers. J. Wind Eng. Indust. Aerodyn. 74-76, 209-218 (1998)

225. Zilitinkevich, S. S., A. A. Grachev, and C. W. Fairall: Scaling reasoning and field data on the sea surface roughness lengths for scalars. J. Atmos. Sci. 58, 320-325 (2001)

226. Zilitinkevich, S., A. A. Grachev, and J. C. R. Hunt: Surface frictional processes and non-local heat/mass transfer in the shear-free convective boundary layer. In: Buoyant Convection in Geophysical Flows, ed by E. J. Plate et al., (Kluwer Acad. Publ., Dordrecht, etc., 1998) pp 83-113

227. Zilitinkevich, S., V. M. Gryanik, V. N. Lykossov, and D. V. Mironov: Thirdorder transport and non-local turbulence closures for convective boundary layers. J. Atmos. Sci. 56, 3463-3477 (1999)

228. Zilitinkevich, S. S., and D. V. Mironov: Theoretical model of thermocline in a freshwater basin. J. Phys. Oceanogr. 22, 988-996 (1992)

229. Zilitinkevich, S., and D. V. Mironov: A multi-limit formulation for the equilibrium depth of a stably stratified boundary layer. Boundary-Layer Meteorol. 81, 325-351 (1996)

230. Zilitinkevich, S. S., V. L. Perov, and J. C. King: Calculation of turbulent fluxes in stable stratification in numerical weather prediction. HIRLAM Newsletter, No. 37, 83-92 (2001)

231. Zilitinkevich, S. S., V. L. Perov, and J. C. King: Near-surface turbulent fluxes in stable stratification: calculation techniques for use in general circulation models. Quart. J. Roy. Meteorol. Soc. 128, 1571-1587 (2002) 



\section{Index}

absolute temperature, 26

boundary-layer approximation, 18

clipping operation, 20, 44

closure assumptions, 7

convective boundary layer, 12, 14, 15, $20,21,47$

convective mass flux, 33

COSMO model, 19, 20

Deardorff convective scaling, 21

deep convection, 3, 29, 31, 44, 49

departure-from-isotropy tensor, 9, 17

detrainment rate, 31

dissipation rates, $7,15,18$

down-gradient approximation, 5, 11

dry static energy, 30

ECMWF IFS, 19, 30, 31

entrainment rate, 31

GME, 19

HIRLAM, 19

large-eddy simulation, 1

liquid water potential temperature, 14, 25,44

liquid water specific humidity, 26, 28

mass-flux approximation, 30

mass-flux convection scheme, 29, 30

Mellor-Yamada closures, 17, 48

Millionshchikov hypothesis, 12, 45
Millionshchikov hypothesis, generalised, 15,46

Monin-Obukhov similarity theory, 21, 22

numerical weather prediction, 1-4, 11, $18,19,29,30,47$

Obukhov length, 23

one-equation turbulence model, 18

organised detrainment, 31

organised entrainment, 31

orographic drag, 2

planetary boundary layer, 1, 3, 16, 23, 25,48

potential temperature, $6,22,26$

potential-temperature skewness, 13

pressure gradient-temperature covariance, 9

pressure redistribution, 8

pressure transport, 11

pressure-scalar covariances, 7, 18

pressure-velocity covariances, 7,18

probability distribution function, 14 , $27,28,43,45,46$

realisability, $8,10,15,20,46$

Reynolds stress, 6

Rotta return-to-isotropy parameterisation, 8

roughness length, 23

scalar flux, 6 
scalar flux, mass-flux approximation, 40 scalar variance, $6,44,47$

scalar variance, mass-flux approximation, 36

SGS cloud scheme, 28, 29, 44

shallow convection, $3,25,29$

specific humidity, 6, 22, 30

stably stratified boundary layer, 19, 22, 25

sub-filter scale, 2,5

sub-grid scale, 2, 3, 5, 20, 34

third-order moments, 5, 11, 21, 33, 45, 46

three-delta-function mass-flux framework, 30, 33, 35

total water specific humidity, 14, 25, 29, 44 truncated closure, 17, 19, 20, 24, 45

turbulence kinetic energy, 2, 7, 20, 25, $42,43,47$

turbulence potential energy, 47

turbulent detrainment rate, 31,41

turbulent entrainment rate, 31,41

two-delta-function mass-flux framework, $14,30,32,33,35,43$

two-equation turbulence model, 18

unified turbulence-shallow convection scheme, 43, 46

vertical-velocity skewness, 13, 34

vertical-velocity variance, mass-flux approximation, 37

virtual potential temperature, $7,21,26$ 
